\title{
Sustainable Polymers Square Table
}

28 \& 29 April 2021

\section{Final Report submitted 24 July 2021}

\section{Organizers/Leaders}

Prof. Juan de Pablo, University of Chicago

Prof. Marc Hillmyer, University of Minnesota

\section{Overview}

The discovery, development, and deployment of sustainable polymers, the molecules that make up plastics, are critical to the health of the globe and its inhabitants. Moreover, the economic vitality of the multitudinous industries that use polymers in their processes and products will be critically reliant on sustainable solutions because of many interwoven factors that include environmental stewardship, policy compliance, and societal and investor demands. Stakeholders in the polymeric materials ecosystem clearly recognize the significant motivating factors in a push to meet the requirements of a circular economy and thus a sustainable future. Inertia associated with the current polymer production and consumption landscapes, however, has resulted in resistance toward change that has proven challenging to overcome. Factors that have contributed to the status quo include the ubiquitous nature of plastics utilization across all sectors of the economy, the incredibly diverse and tunable functional attributes of polymeric materials that make them desirable to other material alternatives, and the highly optimized and economic mass manufacturing capabilities associated with plastics derived from low-cost petroleum resources. There is an urgent need for change to solve our pressing plastics predicament: modern society depends on these polymeric materials and continually expects increased performance and functionality but suffers dire consequences from the associated pollution, which takes the forms that include greenhouse gas emissions from plastic production, and accumulation of plastic waste in the environment. A solution to this polymer puzzle is imperative.

Given the current advantages of plastics in modern society and the clear recognition of the everaccumulating waste associated with their disposal, lack of efficient recycling technologies and infrastructure, and persistence in the environment, it is clear that a new approach is needed We are motivated by the successes associated with the Materials Genome Initiative (MGI), ${ }^{1}$ where the close coupling of experiment and theory has facilitated materials discovery in some sectors. But while an MGI approach has been effective at accelerating the research cycle of prediction, synthesis, and characterization, efforts to incorporate the elements of translation and technological deployment into that cycle have been limited. This is especially true in the soft materials arena. If a next-generation MGl-like approach spanning length scales (from atomic to molecular to material) and time scales (from nanoseconds to decades) can be envisioned, implemented, and supported, and if attention is given to technological feasibility, it will serve as

\footnotetext{
${ }^{1}$ https://www.mgi.gov/about (accessed 19 July 2021)
} 
a platform and guiding light to the materials community as a new approach aimed at addressing crucial societal needs such as polymer sustainability. To help accomplish this feat, the NSFsponsored Square Table brought together a diverse cohort of stakeholders to develop broadranging, integrated, and futuristic ideas and strategies to solve this dilemma in new and innovative ways. The Square Table included emerging and established leaders from a wide range of backgrounds and institutional types such that a clear picture of the key challenges and needs were developed.

Over the course of two half-day sessions, a group of more than 50 participants (see Appendix 1) discussed strategies for advancing the science, technology, and more overarching systems associated with sustainable polymers. The Square Table concept provides structure and selffacilitated interactions among stakeholders who do not typically interact with one another to bridge gaps that exist between industry, federal agencies, and academia in an open and unbiased manner. This was accomplished by mixing participants in breakout discussion with guiding questions, followed by reporting back to the group and repeating with a new mix of participants, questions, and discussion points. Select participants that not only contributed to the discussion but also acted as scribes for sub-sessions were essential to help capture the sum of the views, suggestions, and inputs from the participants. In this way, the discussions cut to the root cause of successes and opportunities in a rapid and less biased manner than traditional roundtables or workshops.

The Square Table objectives included developing proposed answers to key questions such as (1) What are the critical basic and applied questions that, when addressed together, could improve the possibility for impact in terms of moving fundamental research thrusts and translational efforts? (2) What prevents large-scale, coordinated efforts between industry, academia, and federal agencies? (3) What is needed to help reduce these barriers in terms of data resources, physical infrastructure, and/or workforce training? (4) How can data and advanced modeling approaches be shared in a collaborative way that would allow data-driven science and engineering to be accelerated in a translational sense? (5) What partnerships are needed to foster an environment/culture to realize more sustainable polymer solutions in the future?

These discussion questions feed directly into the overarching outcomes for the Square Table. Namely, we have: defined new collaborative research approaches and research partnership models that accelerate discovery in polymers research by combining theory, computation, experiment, and data analytics; addressed community strategies to develop data sharing mechanisms and best practices to support these approaches; identified challenges faced by academia, federal laboratories, and industry in developing partnerships around sustainable polymers; articulated priorities for the potential programs, tools, and services needed to support these partnerships such that translation of sustainable polymers will occur rapidly and at the scale that is needed; developed frameworks for sharing data and accessing shared physical infrastructure and technical resources. In sum, we present a clear picture of the current landscape of sustainable polymers, the key areas of interest moving forward, and the opportunities to harness the vision of advanced translation, innovation, and partnership aspects 
in this arena. This report aims to inform the National Science Foundation on the development of new initiatives that will address the challenges associated with a sustainable polymer future.

Through iterative discussions, critical questioning, and potential solution development, we settled on six topical sessions that focused on distinct but interrelated areas. We worked in the Square Table to refine and crystallize these essential topics and then aimed at presenting creative and innovative solutions and approaches through a variety of "how might we" questions in the following topical areas:

(1) Integration of modern computational approaches and experiments to achieve polymer sustainability for a circular economy

(2) Research and development frameworks supporting translation to circular sustainable polymer systems

(3) Incentivizing and integrating translational processes and practices across sustainable polymer stakeholders

(4) Data best practices to accelerate discovery and translation of sustainable polymers

(5) Sustainable polymer communication, policy, and education through the development of universal language and shared understanding

(6) Societal adoption of sustainable polymers manufactured for in-use performance and circularity in terms of triple bottom line thinking: people, profit, planet

In the following sections, we expand on each of these topical areas and conclude each with key takeaway messages. Sustainability is an issue that is linked to many opportunities, sectors, and to multitudes of opportunities over vast scales. Here, we focus on polymer sustainability with an aim to identify current challenges that keep sustainable polymers from becoming everyday items. Moreover, we aim to provide potential insights into mechanisms and means by which to bring about change such that these opportunities are addressed in as rapid of a manner as possible. 


\section{(1) Integration of modern computational approaches and experiments to achieve polymer sustainability for a circular economy.}

Theory and computation will play a significant role in the quest to make polymeric materials more sustainable. Modern simulation approaches permit exploration of a vast parameter space at a fraction of the cost of the corresponding experimental resources (e.g., solvents, precursors, catalysts, and monomers). They also allow for rapid screening of new sustainable polymer candidates and their corresponding properties. The latter aspect will become particularly important as machine learning opportunities gain ground in the field of polymer design. It is important to emphasize, however, that while the combination of machine learning and computer simulations is enabling much more efficient and expansive screening of new materials, the effectiveness of machine learning methods depends on access to large data sets. ${ }^{2,3}$ Such sets can be generated using simulation and automated experimentation. ${ }^{4,5}$ Designing new materials in this manner will enable rapid prototyping, faster than with experiments alone, and with the urgency that is necessary to arrive at sustainable solutions.

(1a) How might we advance theory, simulation, and computation so that they become more effective for the discovery, development, and translation of sustainable polymers?

Current computational models can consider most organic compounds as potential building blocks for polymeric materials. At present, however, not all relevant time and length scales are easily accessible. Properties that are determined by short time (ns) and short length scale (atomic) processes (e.g., density or enthalpy) can be predicted with established force fields and available simulation tools. To do so, one must rely on algorithms that permit relaxation of polymer conformations, and such algorithms are not generally available in standard simulation tools. Furthermore, many properties of interest (e.g., diffusion or viscosity) depend on molecular processes that occur over time and length scales that are well beyond this limited range. Recent work suggests that hierarchical coarse graining could cover the wide spectrum of scales that is characteristic of polymeric materials, but more effort is needed to gauge the general validity and applicability of such a strategy. ${ }^{6}$ For this thrust, it is particularly important to develop hierarchical modeling frameworks that not only preserve thermodynamics from one level of description onto another, but also kinetic properties. Note that polymeric materials derive some of their benefits

\footnotetext{
${ }^{2}$ Kumar, J. N.; Li, Q.; Jun, Y. "Challenges and opportunities of polymer design with machine learning and high throughput experimentation" MRS Communications 2019, 9, 537-544. https://doi.org/10.1557/mrc.2019.54.

${ }^{3}$ Chen, G.; Shen, Z. Q.; Iyer, A.; Ghumman, U. F.; Tang, S.; Bi, J. B.; Chen, W.; Li, Y. "Machine-Learning-Assisted De Novo Design of Organic Molecules and Polymers: Opportunities and Challenges." Polymers 2020, 12. https://doi.org/10.3390/polym12010163.

${ }^{4}$ Meier, M. A. R.; Hoogenboom, R.; Schubert, U. S. "Combinatorial methods, automated synthesis and highthroughput screening in polymer research: The evolution continues." Macromolecular Rapid Communications 2004, 25, 21-33. http://doi.org/10.1002/marc.200300147.

${ }^{5}$ Trobe, M.; Burke, M. D. "The Molecular Industrial Revolution: Automated Synthesis of Small Molecules." Angewandte Chemie-International Edition 2018, 57, 4192-4214. http://doi.org/10.1002/anie.201710482.

${ }^{6}$ Zhang, G. J.; Chazirakis, A.; Harmandaris, V. A.; Stuehn, T.; Daoulas, K. C.; Kremer, K. "Hierarchical modelling of polystyrene melts: from soft blobs to atomistic resolution." Soft Matter 2019, 15, 289-302. http://doi.org/10.1039/c8sm01830h
} 
from unique rheological and mechanical characteristics, which are inaccessible without correctly modeling their dynamics even in highly coarse-grained representations. Importantly, such characteristics are often the result of a particular set of processing conditions or history, serving to underscore the need for approaches capable of predicting the response of a material to a particular series of processing steps. Recent efforts have started to develop multi-scale approaches capable of accessing time scales that allow the relaxation of polymers with industrially relevant molar masses but have thus far been limited to equilibrium situations. ${ }^{7}$

For sustainability efforts, however, available simulation strategies are still insufficient. Research into new models that incorporate aspects of viable synthetic pathways is necessary to facilitate the study of the full life cycle of newly proposed polymeric materials. ${ }^{8}$ Only if an economically viable synthesis pathway is accessible can the material be considered for industrial adoption. It is therefore important to develop methods that can predict such pathways early in the material discovery-during or before evaluation by computer simulations. It is also important to develop models for computer simulations that can estimate the degradation characteristics of materials. This can include both pathways for recycling after use, as well as any "natural" degradation due to environmental conditions in, for example, landfills or for improperly disposed materials. Degradation is generally a result of chemical changes, usually the scission of covalent bonds, that naturally occur on time scales of months or years, well out of range of current models. Research is thus needed to develop models that consider synthesis and degradation for realistic equilibrium and non-equilibrium polymer conformations and environments, thereby arriving at a computational materials design strategy that integrates the full life cycle from cradle to graveessential for sustainable materials - at the early discovery stages. A critical related aspect is that of incorporating considerations of translation into the computational design process. Such considerations include availability and cost of raw materials, ease of synthesis, ease of processability, and safety concerns. Figure 1-1 presents a schematic view of how simulations can advance a product design. It is possible to gain insights from simulations into the final product capabilities, which allows simulation-driven development cycles. However, it is apparent that current simulation techniques are unable to predict several properties of the late product life, including degradation and recycling. Without improvements here, these aspects cannot be included in the early stages of design and enable development of materials optimized for a circular life cycle.

\footnotetext{
${ }^{7}$ Behbahani, A. F.; Schneider, L.; Rissanou, A.; Chazirakis, A.; Bacova, P.; Jana, P. K.; Li, W.; Doxastakis, M.; Polinska, P.; Burkhart, C.; Muller, M.; Harmandaris, V. A. "Dynamics and Rheology of Polymer Melts via Hierarchical Atomistic, Coarse-Grained, and Slip-Spring Simulations." Macromolecules 2021, 54, 2740-2762. http://doi.org/10.1021/acs.macromol.0c02583.

${ }^{8}$ Zhang, D. D.; del Rio-Chanona, E. A.; Shah, N. "Screening Synthesis Pathways for Biomass-Derived Sustainable Polymer Production." ACS Sustainable Chemistry \& Engineering 2017, 5, 4388-4398. http://doi.org/10.1021/acssuschemeng.7b00429.
} 


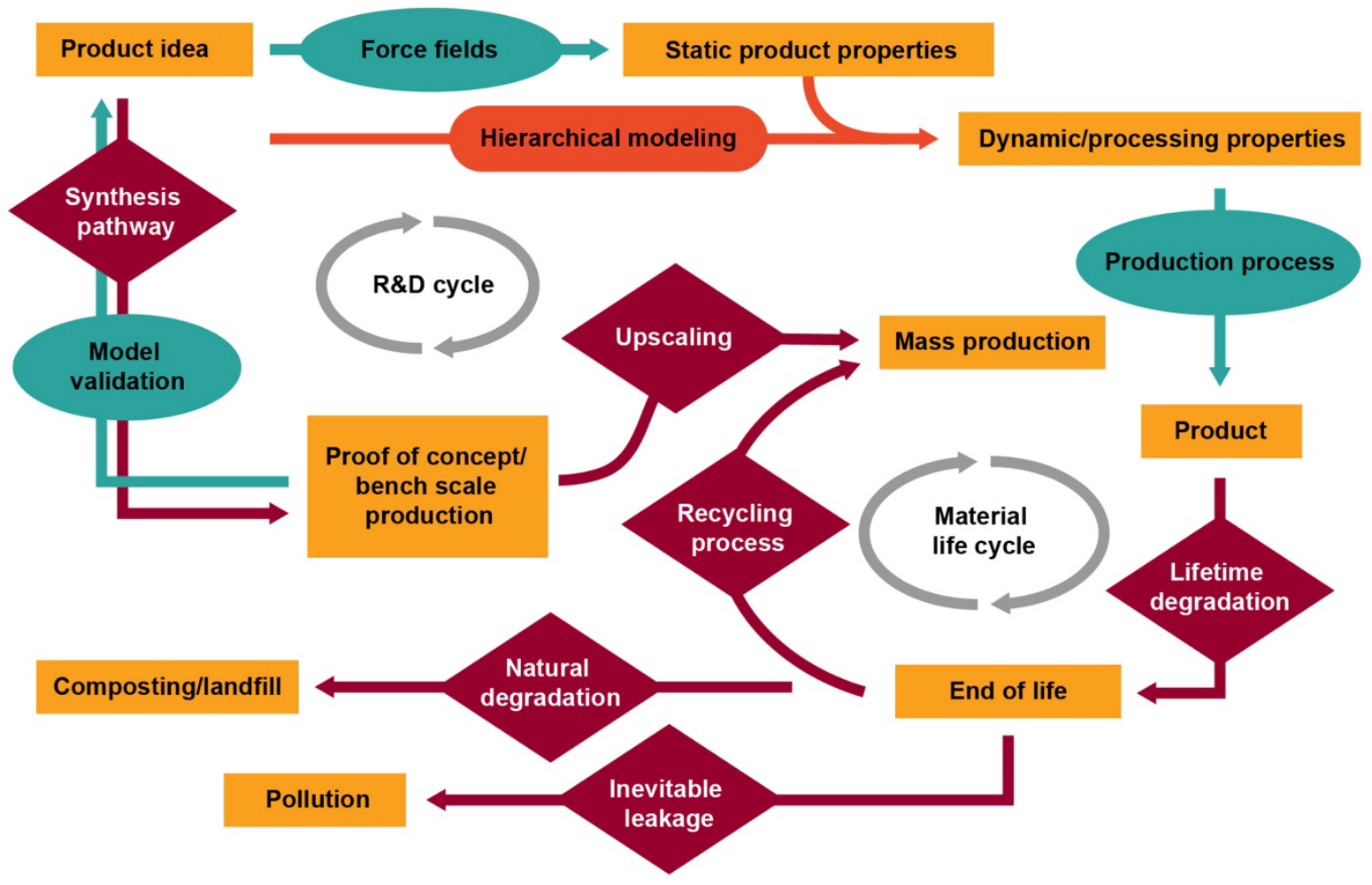

Figure 1-1. Impact of simulation on the life and development of polymeric materials. The arrows indicate how simulations can help to design the material. Green signals that it is possible now, yellow processes possible for specific cases but not in general, and red are processes with few specific or no models widely available for predictions. Overall, simulation can help to design new materials of the production half of the cycle but is lacking for the end-of-life properties, including degradation and recycling. Hence, new models must be developed for the late product stages to close the loop.

(1b) How might we more closely couple experiment with computation and exploit resultant synergy to accelerate advances, discovery, and translation?

An important limitation of computer simulations is the accuracy of the underlying models. Assessing this accuracy is essential if calculated properties are to be used in decision-making for all stakeholders. It is mathematically possible to incorporate automatic accuracy estimates into models, for example with Bayesian regression models in machine learning applications, but the most essential component remains validation against experimental measurements. In general, some experimental data are available, but their usability for simulation model validation varies dramatically-see Section 4 for a more detailed discussion on data exchange. Two important hurdles must be overcome: (i) communication barriers between experimental and simulation researchers and (ii) lack of incentive for experimental researchers to produce validation data. The communication barrier is driven by the often-differing backgrounds of experimental scientists and the researchers that develop and implement state-of-the-art simulation models. Both paths are highly specialized, and it is challenging to cross train scientists and engineers early in their 
careers without compromising a necessary high level of expertise in the respective fields. It is desirable to implement new programs that aim for combined education, which will help bridge language barriers between different players, but experts that pursue a highly focused career in their respective specialties will still be necessary. An alternative approach may be to create external incentives to form teams with members proficient in both approaches, not only as traditional collaborations between but also within research groups.

The second barrier is more entrenched. There are few incentives for an experimental research group to produce dedicated validation data for simulation model building or for machine learning, even as it may be essential for new model or method development. This kind of research usually requires repeated experiments, with many similar but slightly different chemistries, and it involves substantial efforts. It is not the type of research that necessarily drives new scientific discoveries, and it does not garner the recognition from the scientific community that other endeavors do. It would therefore be important to introduce other incentives, including funding, for research groups or companies that generate the much-needed high-quality data for model validation. Interdisciplinary centers could play an important role in this context, where the copublication model would help increase the impact of validation data.

Additionally, investment into fully automated experimentation workflows could accelerate data generation. With anticipated large investments in sustainability, manufacturers of robotic systems could be encouraged to integrate automated workflows, which could then deposit the generated data automatically into open-source data banks. As a result, publicly funded investment would guarantee that the data are accessible to a large audience, and not only select groups, thereby creating a potentially competitive environment for research. Furthermore, such an automatic workflow would necessarily establish standards about formats and meta-data that may be adopted by other stakeholders due to their common availability.

(1c) How do we encourage and facilitate the adoption of theory and computation for the design and translation of polymeric materials, including software development?

Simulation software tools are often developed and maintained by highly specialized researchers focused on their research projects; software development is streamlined for individual projects (or research groups). The scientific peer-reviewing system is in place to ensure that published simulation data are reliable. However, the quality of the codes that generate such data is not generally ensured, and codes are not generally included in publications. Additionally, materials scientists typically receive little education in software engineering. As a result, scientific programs are usually not optimally developed, further complicating maintenance and transferability to future projects. This problem has been recognized before, and initiatives have been launched to address it, ${ }^{9}$ but currently there are few incentives for researchers to devote their efforts to the "quality" of a code. Career-advancing recognition is tied to scientific publications, as opposed to the elegance of a simulation code. There are examples of well-maintained open-source projects,

\footnotetext{
${ }^{9}$ See for example: https://bssw.io/ (accessed 19 July 2021)
} 
for example for molecular dynamics simulations (e.g., LAMMPS). ${ }^{10}$ Still, better solutions are needed; it is critical that new software projects be supported for long periods, such that the community can rely on them for long-term planning. Incentives for entrepreneurship or the establishment of dedicated career paths for support of software projects are potential solutions. We explicitly note that open-source and entrepreneurship are not mutually exclusive in this context, as successful business models for paid support like the Red Hat ${ }^{11}$ or Ovito ${ }^{12}$ projects have demonstrated, but large user bases are needed for such approaches to be viable. Extended support for scientific software is expected to play an important role in the future. To intensify computational research for sustainable materials it is desirable to widen the scientific user base to researchers without a background in computational methods; training and support for this user base should be facilitated. Additionally, it would be desirable to make scientific software more accessible to a broader user base. This should be done while introducing safeguards against involuntary usage with scientifically inappropriate parameters. Such safeguards are necessary to prevent inexperienced users from generating compromised data unsuitable for broader disemination. Providing access to dedicated user support, for example, at national labs or companies, would democratize access to software because new feature requests could be made by users who are not computationally well-versed. Otherwise, new features can only be included if the requesting party brings the expertise and resources to develop and integrate them on their own. In both cases, dedicated support is required to enable such an ecosystem.

Key Takeaways

- Simulations represent powerful and economic tools to develop new polymeric materials.

- Research is required to further develop hierarchical coarse-graining approaches capable of predicting equilibrium and non-equilibrium properties, including the effects of processing, and to extend existing models to include degradation and recycling pathways, thereby incorporating sustainability aspects in prediction and design.

- Accessibility and reliability of software require support for development and maintenance, as well as for career pathways focused on training and connecting researchers with diverse backgrounds to simulation science and technology.

\section{(2) Research and development frameworks supporting translation to circular sustainable polymer systems}

A transition towards a circular plastic economy will be crucial to minimize environmental damage while maintaining the value of plastics. This transition will necessitate systems-level thinking, as plastic products would ideally be durable, high-performing, and produced using sustainable resources, while being able to be reused, repaired, recovered, or recycled at the end of their

10 https://www.lammps.org (accessed 19 July 2021)

11 https://www.redhat.com/en (accessed 19 July 2021)

12 https://www.ovito.org/about/ovito-pro/ (accessed 19 July 2021) 
service life. ${ }^{13}$ To achieve zero waste targets, two main requirements stand out: increasing the recyclability and repurposing of current materials and enabling the production of new materials that can be easily recycled or the redesign of products such that non-recyclable plastics are avoided (e.g., microbeads in personal care products or plastics that contain harmful additives). Bio-based plastics have also been investigated in the context of circular plastics, where the eventual biodegradation or incineration allows the biogenic carbon to be released back into the atmosphere to be sequestered by living biomass taken, thereby closing the loop. ${ }^{14}$ To break the traditional linear economy of produce-use-discard, both technological and policy innovations are needed. Fundamental scientific understanding gaps and technological developments that advance the sustainable landscape should be identified, the full system and life cycle analyses should be included in the design of next-generation polymers, and robust and consistent infrastructure should be built to support circular material systems.

(2a) What are the fundamental scientific understanding gaps, and how might we identify technological developments that fundamentally advance the sustainable landscape?

Identifying the scientific knowledge gaps in designing, producing, and reprocessing plastics is important to provide roadmaps to plan and target future research. Some areas of research critical to achieving a circular system are noted in Figure 2-1. Quantifying the value of closing these knowledge gaps will enable projections on progress towards sustainability targets and provide foresight into the emergence of new materials. This, however, is challenging due to the interconnectedness and interdependence of many knowledge gaps, and it is exacerbated by a lack of information sharing across sectors and the value chain. As discussed in Sections 3 and 4, increased collaboration is essential, and well-maintained databases are needed for structureproperty relationships, life-cycle analysis, and techno-economic analysis. In addition, bridging the gap between policymakers and scientists could lead to a more efficient and effective allocation of resources for research.

\footnotetext{
${ }^{13}$ Bucknall, D. G. "Plastics as a materials system in a circular economy." Philosophical Transactions of the Royal Society a-Mathematical Physical and Engineering Sciences 2020, 378, 2176. http://doi.org/10.1098/rsta.2019.0268

${ }^{14}$ Spierling, S.; Rottger, C.; Venkatachalam, V.; Mudersbach, M.; Herrmann, C.; Endres, H. J. "Bio-based Plastics - A Building Block for the Circular Economy?" 25th Cirp Life Cycle Engineering (Lce) Conference 2018, 69, 573-578. http://doi.org/10.1016/j.procir.2017.11.017.
} 


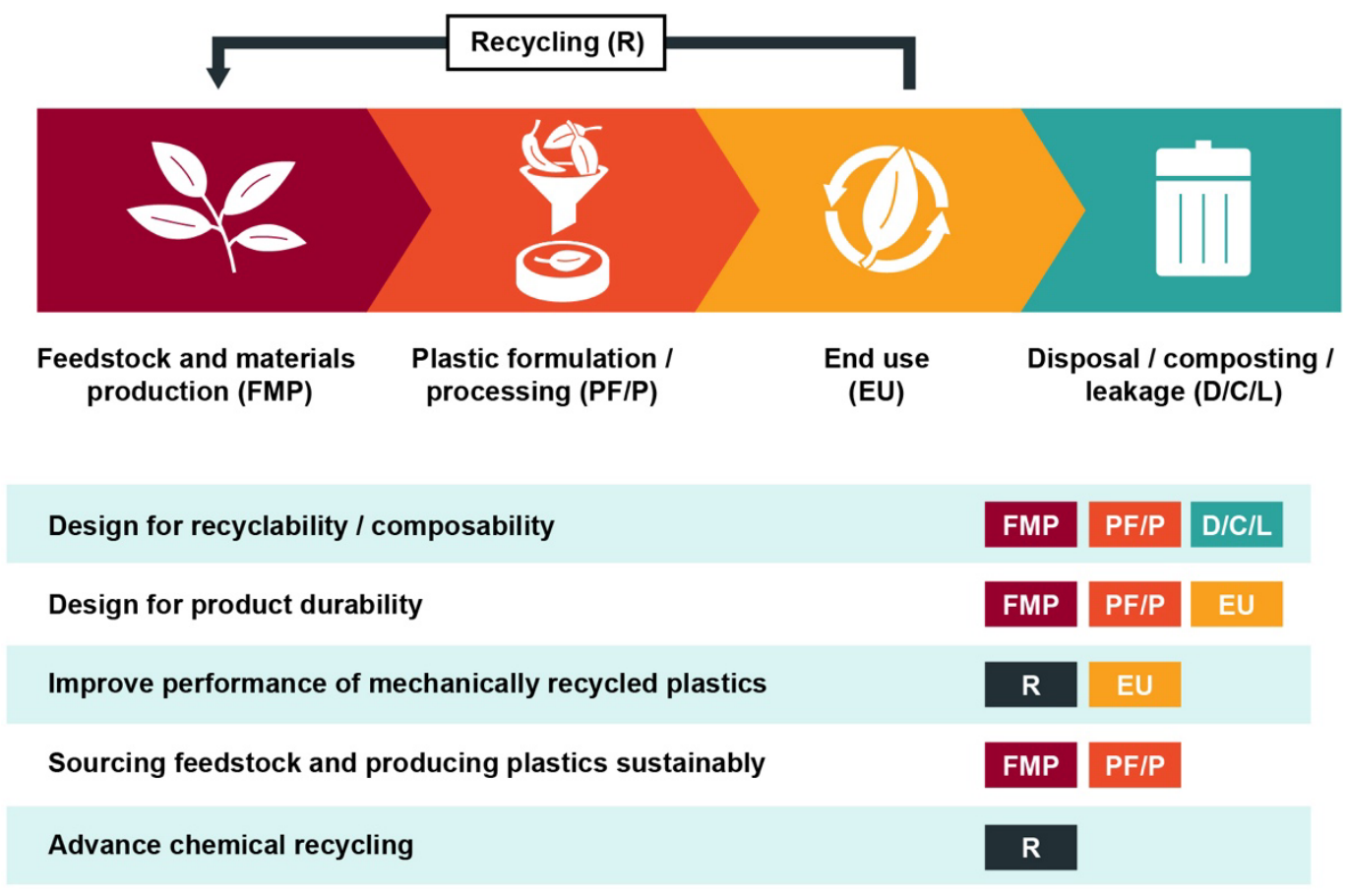

Figure 2-1. Areas of research for advancing plastic sustainability. Various aspects of the plastic life cycle need to be considered in designing processes and products, highlighting the importance of systems-level approaches.

The technical knowledge gap is particularly prominent in addressing complexities in plastic materials at the end of their service life. Recycling is crucial to achieve circularity but is hindered by the diversity of compositions encountered in recycled plastic streams, which are made up not only of different polymers but include multiple additives. Even within the same recyclable polymer class, companies can differentiate their products through polymer molar mass, architecture, processing history, and other characteristics, leading to unavoidable heterogeneities in recyclates. ${ }^{15}$ In addition, the use of certain additives such as opacifiers, dyes, and fillers pose huge challenges to sorting facilities due to incompatibility with near-infraredspectroscopy-based sorting. Plastics containing these additives may contaminate recycled streams if not properly identified. Legacy additives that are still present in older plastics may also pose safety hazards. ${ }^{16,17}$ Even further difficulties arise because plastics may be soiled as a result of use, complicating sorting and adding additional processing requirements. These complexities are compounded by continuously evolving materials landscape and waste targets, necessitating

\footnotetext{
${ }^{15}$ Stein, R. S. "Polymer Recycling - Opportunities and Limitations." Proceedings of the National Academy of Sciences of the United States of America 1992, 89 (3), 835-838. http://doi.org/10.1073/pnas.89.3.835.

${ }^{16}$ Turner, A. "Black plastics: Linear and circular economies, hazardous additives and marine pollution." Environment International 2018, 117, 308-318. http://doi.org/10.1016/j.envint.2018.04.036.

${ }^{17}$ Wagner, S.; Schlummer, M. "Legacy additives in a circular economy of plastics: Current dilemma, policy analysis, and emerging countermeasures." Resources Conservation and Recycling 2020, 158, 104800. https://doi.org/10.1016/j.resconrec.2020.104800.
} 
multifaceted solutions. By taking the complex plastic life cycle into account early in the design phase, one could significantly advance plastic sustainability, for example through new design paradigms for materials with triggerable depolymerization or degradation, and through the redesign of materials without the need for problematic additives or composites while still achieving performance needs.

In addition to addressing the chemical complexity of plastic formulations, innovations in recycling technologies are also required to achieve high-quality recycled products and feedstock. Mechanical recycling can be profitable in certain scenarios, and closed-loop recycling, where the recycled plastic is used in its original application, is feasible for products such as clear PET bottles. However, it can only be implemented for a narrow range of materials and requires relatively pure plastic streams, highlighting the need for improved separation and purification strategies. Further research is also needed to understand potential changes in polymer molar mass and mechanical properties after aging and repeated reprocessing cycles. ${ }^{18,19}$ One strategy to address the diversity in recyclate streams arising from different processing histories may be to develop computational models and machine learning tools (see Section 1), where tailored formulation or processing parameter recommendations can be rapidly provided. Chemical recycling, on the other hand, encompasses a wide range of processes that enable constituent monomers used to prepare the polymers to be recovered and then repolymerized can be an important recycling avenue for plastics containing difficult-to-separate plasticizers and other additives. ${ }^{20}$ More robust methods for depolymerizing a broader suite of materials are needed, along with the development of better catalysts and other low-carbon-footprint strategies for transforming endof-life materials into useful starting materials.

(2b) How might we encourage inclusion of the full system into design and life cycle of the next generation of polymers?

The transition to prioritizing sustainability from the beginning of product design is critical for developing next-generation polymers that are in line with the circular economy. This includes both technical aspects in the basic materials design and economic aspects in the development of new circular business models. For sustainability considerations to be included in design choices, research is required to develop holistic and standardized metrics for assessing circularity and recyclability. In academic settings, funding agencies could encourage more life cycle and technoeconomic analyses to be performed through grant supplements. In addition, early exposure to regulatory and commercialization aspects could provide academic researchers with a broad, systems-level understanding. This offers a link between technical design and practical aspects, for example, by enabling researchers to design recyclable materials based on available

\footnotetext{
${ }^{18} \mathrm{Wu}, \mathrm{G}$. Q.; Li, J.; Xu, Z. M. "Triboelectrostatic separation for granular plastic waste recycling: A review." Waste Management 2013, 33, 585-597. http://doi.org/10.1016/j.wasman.2012.10.014.

${ }^{19}$ Lamantia, F. P.; Vinci, M. "Recycling Poly(Ethyleneterephthalate)." Polymer Degradation and Stability 1994, 45, 121-125. http://doi.org/10.1016/0141-3910(94)90187-2.

${ }^{20}$ Vollmer, I.; Jenks, M. J. F.; Roelands, M. C. P.; White, R. J.; van Harmelen, T.; de Wild, P.; van der Laan, G. P.; Meirer, F.; Keurentjes, J. T. F.; Weckhuysen, B. M. "Beyond Mechanical Recycling: Giving New Life to Plastic Waste." Angewandte Chemie-International Edition 2020, 59, 15402-15423. http://doi.org/10.1002/anie.201915651.
} 
infrastructure. Over-emphasizing those practical considerations could potentially inhibit or stifle research, and it should therefore be balanced with the benefits. Policies should be developed to incentivize companies to consider life cycle in addition to profitability or financially motivate the design of products that are more recyclable and sustainable. This may be achieved through extended producer responsibility schemes, in which fees can be levied to place a price on externalities and shift the cost of disposal onto manufacturers. Implementable policies that are successful at achieving circular economy objectives require policymakers to be informed on best practices and have the means to evaluate the effectiveness of such policies (see Section 5). Therefore, increasing routes for scientists to work directly with policymakers, for example, through internships or fellowships supported by institutes and universities, could be highly beneficial for both parties. In addition, partnerships with policy experts through, for example, non-profit entities that provide transparency to consumers regarding issues of sustainability would be a complementary driving force for change given the potential power of consumer sentiment.

The market entry of novel sustainable plastics will greatly benefit from the integration of sustainability with business development. Transition to a circular economy entails shifts in business models and new ways of thinking about delivering value and resource management. Potential new models that have gained attention include product-service systems, where businesses retain ownership of products while providing the use or function of the product as a service. ${ }^{21}$ Symbiotic business models, with greatly facilitated exchange of materials between companies, have also been explored, particularly when one company's waste is used as another's raw material. However, new circular economy models can be challenging to implement due to barriers such as risk aversion, technical challenges in product quality, lack of understanding of how such a transition would generate revenue, and dependencies on other actors in the supply chain. ${ }^{22}$ In addition, the transition will inevitably generate opportunities for disruptive technologies, which might threaten incumbents who could resist these new changes. Research on how to develop strategic business models and address barriers to adoption will be crucial in the global perspective of speeding up this transition. The need for the value chain to be aligned around sustainability issues also requires more collaboration and shared responsibility amongst stakeholders, including polymer producers, compounders, collectors, sorters, recyclers, and composting facilities. Financial incentives and frameworks for interdisciplinary collaboration may be needed, ${ }^{23}$ although market forces and investor demand alone may also drive businesses to innovate in the direction of circularity and sustainability. ${ }^{24}$ In this regard, transparent policies would be beneficial as they allow companies to make decisions regarding product sustainability.

\footnotetext{
${ }^{21}$ Ferasso, M.; Beliaeva, T.; Kraus, S.; Clauss, T.; Ribeiro-Soriano, D. "Circular economy business models: The state of research and avenues ahead." Business Strategy and the Environment 2020, 29, 3006-3024. http://doi.org/10.1002/bse.2554.

22 itzen, S.; Sandstrom, G. O. "Barriers to the Circular Economy - integration of perspectives and domains." 9th Cirp Industrial Product/Service-Systems (Ipss) Conference: Circular Perspectives on Product/Service-Systems 2017, 64, 7-12. http://doi.org/10.1016/j.procir.2017.03.005.

${ }^{23}$ Esposito, M.; Tse, T.; Soufani, K. "Is the Circular Economy a New Fast-Expanding Market?" Thunderbird International Business Review 2017, 59 (1), 9-14. http://doi.org/10.1002/tie.21764.

24 https://www.bloomberg.com/news/articles/2021-05-03/dupont-loses-plastic-pollution-vote-with-record-81-rebellion (accessed 19 July 2021)
} 
(2c) How might we streamline a robust and consistent infrastructure to enable full plastic circularity?

A robust infrastructure for supporting plastic circularity is essential for adoption of new sustainable practices and materials that consider full supply chain effects. Recruiting diverse researchers to bring fresh perspectives to the recycling problem and developing frameworks that support partnerships between academics and industry is important for the successful commercialization of new materials technologies.

Current low volumes of plastic recycling have been partly due to the lack of favorable public policies, incentives, and regulations that promote the economic feasibility of recycling practices (see Section 5). The infrastructure for collecting and sorting end-use products is also inadequate for processing heterogeneous waste streams and is a particular issue for composites and newer materials. Therefore, scientifically informed policies are needed to drive investment into infrastructure. This requires research into methods and models to develop and evaluate the effectiveness of policies, including the various collection and extended producer responsibility schemes. ${ }^{25,26}$ Frameworks that promote collaboration among multiple parties in the plastic value chain may also play a role in boosting the collection of after-use plastics through streamlined material exchanges and the reduction of recycling incompatibility. One barrier identified in this area is the need for a cohesive and comprehensive strategy to achieve plastic circularity. In addition, policies can often have implications on global trade markets, complicating situations in which sustainability targets conflict with other objectives.

Key Takeaways

- Research efforts are needed to address the diverse compositions and complexities for recycled plastic streams to remove technical barriers to recycling.

- Plastic life cycle, circularity, carbon footprint, and commercialization aspects should be considered early in the design phase in the development of next-generation polymers.

- Transparent policies informed by science and collaborative frameworks that include experts from STEM, social, economic, and business, are needed for driving the transition to circular materials platforms.

\section{(3) Incentivizing and integrating translational processes and practices across sustainable polymer stakeholders}

As mentioned above, the plastics ecosystem is enormous and includes a wide array of stakeholders, such as suppliers, producers, manufacturers, consumers, waste managers,

\footnotetext{
${ }^{25}$ Nash, J.; Bosso, C. "Extended Producer Responsibility in the United States Full Speed Ahead?" Journal of Industrial Ecology 2013, 17, 175-185. http://doi.org/10.1111/j.1530-9290.2012.00572.x.

${ }^{26}$ Hanisch, C. "Is extended producer responsibility effective?" Environmental Science \& Technology 2000, 34, 170a175a. http://doi.org/10.1021/es003229n.
} 
communities, investors, and governments. The transition to a sustainable polymer economy will require all these entities - along with their diverse (and often competing) interests - to work in concert towards common and well-defined goals. To do so, some key challenges must first be addressed. Here we identify three crucial questions in this domain: (i) how to design and implement clear and useful measures of sustainability, (ii) how to develop a "roadmap" for the polymer lifecycle that enables cooperation between various stakeholders, and (iii) how to connect organizations and communities and facilitate communication between them more effectively to accelerate innovation.

(3a) How might we generate sound sustainability metrics from environmental, social, and governance perspectives and with a target of universal agreement among the broad distribution of stakeholders?

Although materials scientists have long been able to effectively measure and quantify polymer material performance, analogous measures of polymer sustainability have proven elusive (see Section 6). This is in part due to the breadth of the problem. For example, the term "sustainable" may have very different connotations for researchers vs. consumers vs. investors (see Section 5). ${ }^{27}$ Without clear and quantifiable metrics that all stakeholders can agree upon, different communities, countries, and economies may needlessly replay the same debates. Furthermore, other scientists, policymakers, and industry groups may focus on portions of the plastics economy that are not necessarily reliable determinants of sustainability. If robust and transparent sustainability metrics can be developed, they will help improve confidence in policymaking and ensure that efforts to address polymer sustainability take a complete view of the challenge. Such metrics would also allow product developers to consider sustainability from the beginning of the design process, rather than as an afterthought, leading to fundamental change in the plastics economy.

One of the key hurdles in developing standardized sustainability metrics is the limited availability, reliability, and transparency of data covering the full lifecycle of polymer materials and across diverse organizations. Polymer materials are not easily traceable from synthesis through manufacturing, distribution, use, and disposal, with significant data gaps regarding end-of-life behavior. ${ }^{28,29}$ These issues of data availability prevent researchers from accurately quantifying the effect of materials supply chains on societal wellbeing.

To address these challenges, and as noted earlier, a wide range of interdisciplinary research is required; closer collaborations are needed between polymer scientists, engineers, economists,

\footnotetext{
${ }^{27}$ Williams, C. C.; Millington, A. C. "The diverse and contested meanings of sustainable development." Geographical Journal 2004, 170, 99-104. http://doi.org/10.1111/i.0016-7398.2004.00111.x.

${ }^{28}$ Thompson, R. C.; Moore, C. J.; vom Saal, F. S.; Swan, S. H. "Plastics, the environment and human health: current consensus and future trends." Philosophical Transactions of the Royal Society B-Biological Sciences 2009, 364, 21532166. http://doi.org/10.1098/rstb.2009.0053.

${ }^{29}$ Heller, M. C.; Mazor, M. H.; Keoleian, G. A. "Plastics in the US: toward a material flow characterization of production, markets and end of life" Environ. Res. Lett. 2020, 15, 094034. https://iopscience.iop.org/article/10.1088/1748-9326/ab9e1e/meta
} 
social scientists, environmental experts, and policymakers. For instance, capturing and quantifying the end-of-life fate of polymers involves polymer chemistry, environmental science, and regulatory expertise. Similarly, life cycle analysis (LCA) will benefit from new data sources that reflect polymer-specific properties and manufacturing practices. ${ }^{30}$ Social scientists and economists can play a key role by identifying how organizations, entities, and consumers make decisions within the plastics economy and modeling or predicting how various policy scenarios might affect diverse stakeholders. Case studies on related sectors and certification programs (e.g., LEED, ${ }^{31}$ Fair Trade, ${ }^{32}$ Sustainably Forested ${ }^{33}$ ) are also needed to ensure that common issues and pitfalls can be avoided. ${ }^{34,35,36}$

In addition to aggressive research in this area, additional infrastructure is also needed. First, a well-maintained database is required that includes not only physical property data, but also polymer-specific environmental persistence, mobility, and toxicity data (see Section 4). Second, sustainability certification programs must require data availability and transparency throughout the entire plastics supply chain. Recent technological advances, such as blockchain-based public ledgers may prove valuable in these efforts. ${ }^{37}$ Finally, strong interagency coordination is needed to set industrial and regulatory standards throughout the polymer economy and maintain funding for long-term success.

(3b) How might we create a roadmap for the full life cycle of polymers in which stakeholders work together along the value chain towards common goals in sustainability?

Perhaps the best-known example of community-wide cooperation in research and development is the "International Technology Roadmap for Semiconductors," which for many years has guided the activities of companies, academic researchers, and nonprofit consortia such as the Semiconductor Research Corporation (SRC) ${ }^{38}$ and Sematech. ${ }^{39}$ A similar roadmap would be extremely valuable in the transition to sustainable polymer materials. Such roadmaps help align research efforts, allowing progress to proceed in parallel (rather than sequentially) and assisting various entities to coordinate intellectual property and data accessibility without sacrificing

${ }^{30}$ Ayres, R. U. "Life-Cycle Analysis - a Critique." Resources Conservation and Recycling 1995, 14, $199-223$. http://doi.org/10.1016/0921-3449(95)00017-D.

${ }^{31}$ www.usgbc.org/leed (accessed 19 July 2021)

${ }^{32}$ www.fairtradecertified.org/ (accessed 19 July 2021)

${ }^{33}$ www.fsc.org/en/forest-management-certification (accessed 19 July 2021)

${ }^{34}$ Scofield, J. H. "Do LEED-certified buildings save energy? Not really ..." Energy and Buildings 2009, 41, 1386-1390. http://doi.org/10.1016/i.enbuild.2009.08.006.

35 Booth, P.; Whetstone, L. "Half a cheer for Fair Trade." Economic Affairs 2007, 27, 29-36. http://doi.org/10.1111/i.1468-0270.2007.00727.x.

36 https://e360.yale.edu/features/greenwashed-timber-how-sustainable-forest-certification-has-failed (accessed 19 July 2021)

37 Iansiti, M.; Lakhani, K. R. "The Truth About Blockchain." Harvard Business Review 2017, 95, 119-127. https://hbr.org/2017/01/the-truth-about-blockchain

${ }^{38}$ https://www.src.org (accessed 19 July 2021)

39 Gargini, P. In The International Technology Roadmap for Semiconductors (ITRS):" Past, present and future", GaAs IC Symposium. IEEE Gallium Arsenide Integrated Circuits Symposium. 22nd Annual Technical Digest 2000.(Cat. No. 00CH37084), IEEE: 2000; pp 3-5. http://doi.org/10.1109/GAAS.2000.906261. 
confidentiality. A sustainable polymer roadmap would help coalesce a global community that can include providers, customers, and waste management, thereby accelerating progress towards shared goals (see Figure 3-1). There have been some contributions in this arena that will inform future efforts. ${ }^{40}$
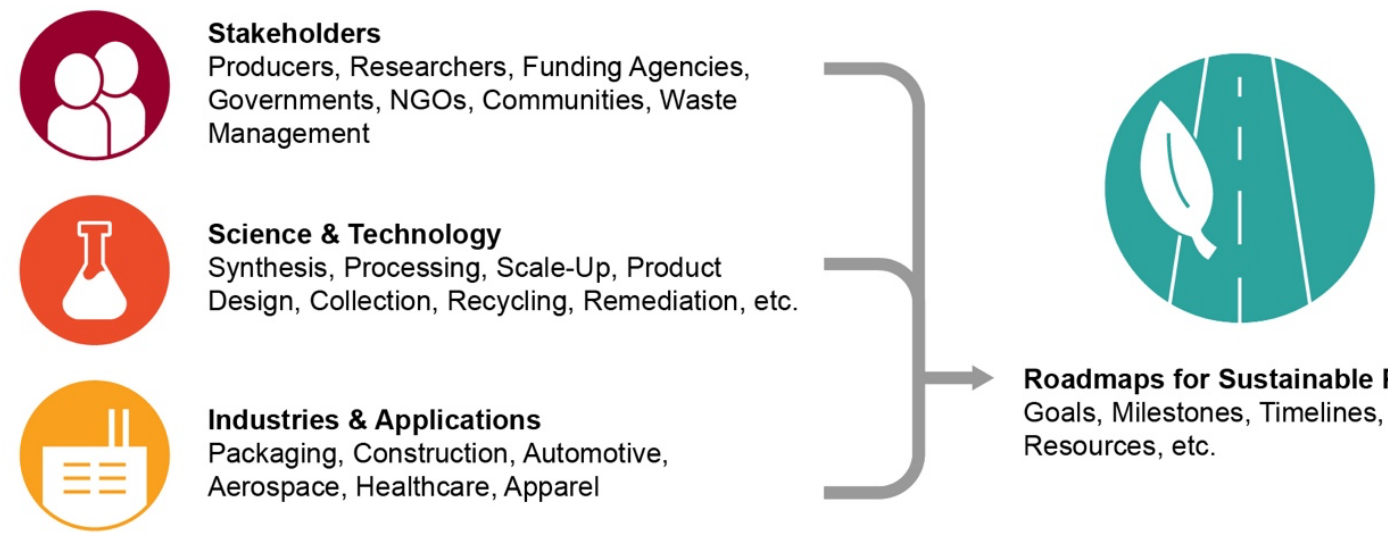

Roadmaps for Sustainable Polymers Goals, Milestones, Timelines, Resources, etc.

Figure 3-1. An example of the inputs (left) and outputs (right) of potential road-mapping efforts for sustainable polymer technologies.

It is important to recognize that while the semiconductor industry offers a useful success story, the plastics economy faces several unique challenges. Multiple sub-roadmaps may be needed depending on the specific applications (e.g., packaging, aerospace, construction, automotive). In addition, the stakeholder community is highly complex with diverse interests. For instance, waste management and consumers do not necessarily participate in product design and development, but these interactions could be important for closing the loop in a circular plastics economy. There will also be differing requirements in the case of single use plastics as compared to durable, reusable, and robust plastics. To enable cooperation between such disparate players, both public and private leadership is required.

To create useful roadmaps, we must understand all the stakeholders in the polymer ecosystem. An assessment and accounting of the entire community is urgently needed, including, for example, municipalities, users, and start-ups, and how they grapple with the scientific and technological challenges detailed throughout this report. This ontology of the plastic supply chain can then be used to identify and resolve conflicting interests and develop LCA and technoeconomic analysis (TEA) tools and methodologies for determining grand challenges in the field. ${ }^{41}$ Moreover, organizations and institutions in the polymer space may already be creating roadmaps

40 For example, see: The Roadmap To Reuse Plastics Solutions for America 2020 https://www.plasticmakers.org/files/0ad2b4b877997c3b91878b785b6e51f821857c2d.pdf (accessed 19 July 2021) ${ }^{41}$ Burk, C. "Techno-Economic Modeling for New Technology Development." Chemical Engineering Progress 2018, $114,43-52$. 
for this purpose, so it is important that communities coordinate closely to develop a single, coherent vision rather than a plethora of competing objectives. It will also be important to study previous road-mapping efforts in other sectors (such as those generated by the Department of Energy $^{42}$ ) to identify gaps and ensure successful adoption among stakeholders.

For a sustainable polymer roadmap to be successful, it must be owned, maintained, and updated by a dedicated overarching organization. For this purpose, a public-private partnership and/or foundation with strong ties to industry and government could provide an ideal solution. In a similar vein, various agencies and industrial partners could cooperate to establish a manufacturing innovation institute that is dedicated to vertical integration and reinvention of polymer materials. ${ }^{43}$ In the United States, these organizations could be funded by federal agencies, including NSF, DOE, DOD, and NIST, and could support research, development, and infrastructure grants that include universities, companies, municipalities, and other stakeholders. Since plastics innovation must be applied globally, the support of funding agencies worldwide will also be critical for a rapid transition.

(3c) How might we spot opportunities, enable understanding, and communicate breakthroughs across industries, universities, research institutions, governments, and the general public to accelerate innovation?

To respond effectively to the plastic pollution crisis, the pace of technological innovation must accelerate, which requires a better understanding of the entire polymer ecosystem from all parties. For example, companies must be able to rapidly translate research progress into commercial opportunities, while researchers must understand the scientific challenges through the entire material lifecycle to identify key problems and make valuable contributions. One barrier in this area is the shortage of talent in the workforce: transitioning towards polymer sustainability will require a new breed of cross-disciplinary scientist, e.g., chemical engineers or materials scientists with expertise in data science or public policy. Developing this workforce requires investment in innovative education programs for interdisciplinary research. This can be achieved through expanded fellowship programs, private industry advising of graduate students, and more frequent and easier movement of scientists between academia and industry. So too must efforts be placed in developing post-graduate workforce education, such that stakeholders currently in the workforce can acquire relevant skills for sustainability.

Public and local communities are also key components of these efforts: consumers must have a basic understanding of polymers to shift habits towards sustainable plastics use. The polymer science/research community can help speed this transition by offering stronger support for FTC green guides and other programs that help consumers make informed decisions. Understanding the principles of effective public communication (e.g., through case studies, training) will be vital

42 www.energy.gov/eere/vehicles/us-drive-partnership-plan-roadmaps-and- accomplishments (accessed 19 July 2021)

${ }^{43}$ www.manufacturingusa.com/(accessed 19 July 2021) 
to the success of these efforts. ${ }^{44}$ Governments and funding agencies also have a role to play as they can act as enablers for local, community-based improvements. For example, by improving communication and organizational infrastructure, support and investment at the federal level can be more effectively funneled to state and local governments, allowing communities to solve local problems and build up domestic capabilities (e.g., manufacturing, collecting, and sorting). We expect this will encourage a citizen science model and can create a feedback loop for the producer for taking extended product responsibility by promoting more transparency.

Key Takeaway Message

- A polymer upcycling and sustainability roadmap is needed to help researchers, manufacturers, users, and policymakers work efficiently towards common goals in a coordinated and constructive manner.

- It is important to understand the scope of the industry and align the wide array of stakeholders towards common goals.

- Consistent, holistic, innovative, and transparent life cycle assessments are needed, and they should be supported with metrics and data that can guide the decision-making.

- Sustained and coordinated stewardship of the problem is required and the steward(s) must be able to engage all parties and incentivize innovation in technology, infrastructure, and education.

\section{(4) Data best practices to accelerate discovery and translation of sustainable polymers}

The rapid growth of sustainable polymer research will be accompanied by a commensurate increase in the quantity of corresponding data generated. How data are generated, managed, and shared to drive and accelerate the discovery and translation of new technologies is one of the key challenges facing the polymer science and engineering community. ${ }^{45}$ Over the past several decades, an enormous amount of polymer data from both basic and applied research efforts has been generated by various sources, including academia, industry, and the public sector, already making their organization and curation a daunting task. Moreover, most of these data remain inaccessible and isolated from further use, especially for the design of sustainable polymers, due to several issues.

First, the key research and data priorities for the field of sustainable polymers remain elusive. Identifying the properties that a sustainable polymer needs to have could guide a better use and further development of existing polymer databases. Second, limited data reliability and consistency present formidable challenges. Collection practices are rarely standardized, and system parameters are often incompletely reported, which stymies efforts to assemble highquality and searchable databases. While data collection and reporting procedures can and must

\footnotetext{
${ }^{44}$ www.ftc.gov/news-events/media-resources/truth-advertising/green-guides (accessed 19 July 2021)

45 Audus, D. J.; de Pablo, J. J. "Polymer Informatics: Opportunities and Challenges." ACS Macro Letters 2017, 6, 10781082. http://doi.org/10.1021/acsmacrolett.7b00228.
} 
be improved, the question of how to handle previously collected data sets remains open. Lastly, the goal of comprehensive databases requires coordinated contributions from academic, industrial, and governmental partners. This raises the question of how to incentivize cross-sector collaboration while managing issues surrounding data sharing amongst multiple entities, both public and private. In this section, we review challenges related to data accumulation, management, and sharing in more detail and assess the work needed to address them (summarized in Figure 4-1).

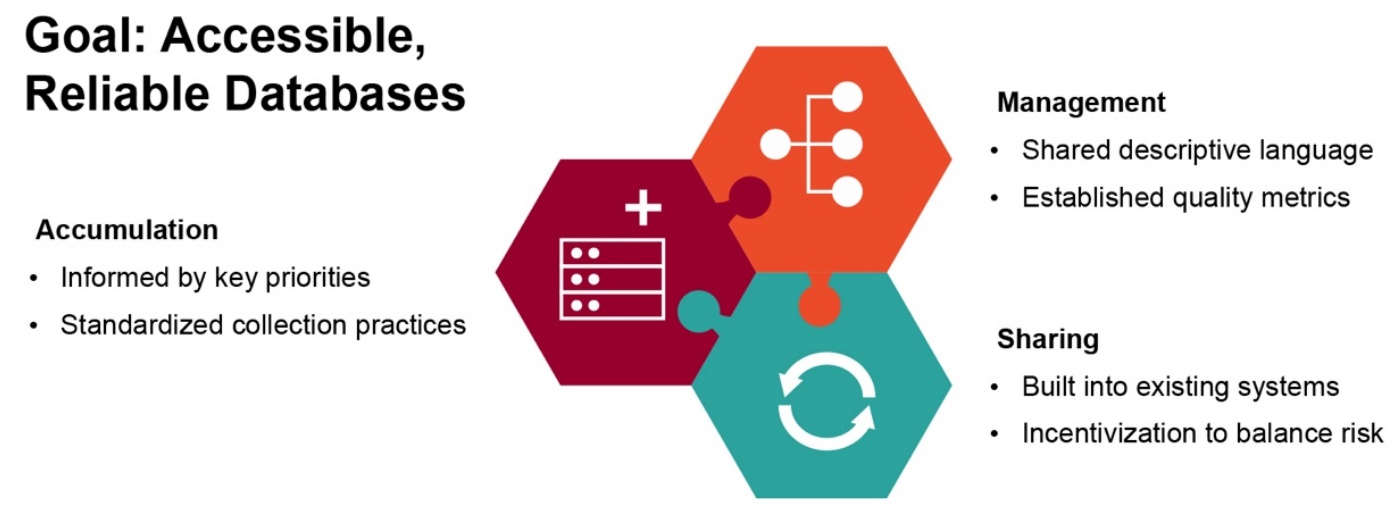

Figure 4-1. The three facets of accessible, reliable databases: accumulation, management, and sharing.

(4a) How might we prioritize the data that are needed to address sustainable polymer grand challenges?

The future of the sustainable polymers field will center on several "grand challenges," which will require accessible data upon which stakeholders including researchers and policymakers can base their decisions. It is essential that we establish clear priorities for which data are most impactful in the interest of sustainability, especially as the limited resources available for this task necessitate a well-defined scope. Yet, the process of data accumulation has been largely driven from the bottom up, with no overarching guidance. Most of the generated data are highly application-specific or quite specialized in nature due to the goals of a given research project. This decentralized data generation process has led to diverse metrics for qualities related to sustainability, including material toxicity, energy input, and recyclability. Consequently, comparison across multiple disparate datasets is, at a minimum, extremely difficult.

To overcome this fragmented data space, unified metrics as well as standards on data collection and description are needed. These metrics should be established for a broad range of polymer properties, including stability, reactivity, and mechanical properties. Although finding a universal metric that everyone agrees upon might be challenging, identifying the sub-fields of polymeric materials, and unifying existent metrics in each sub-field could be an attainable next step. Furthermore, standards should be defined in a descriptive enough manner such that data can be understood when being accessed within a database context. For instance, measurements may 
be taken to address an intended application, but the reported properties should be broadly recognizable. These efforts will require a top-down approach, or the coordination by a professional society or societies, with participation of wide range of data producers.

Beyond standardized metrics, it is essential that the community develops a clearly defined, shared language for the representation of polymer data. This would be the simplest way to guarantee data accessibility at the source, improving later readability by both human and machine. Polymers present a particular challenge to the use of standardized language. For one, the existence of multiple conflicting naming conventions, as well as trade names, presents an obstacle towards a mutual understanding and efficient sharing of data. While efforts have been made towards standardization, such as IUPAC nomenclature, these names tend to be less commonly used-consider polystyrene versus the IUPAC equivalent poly(1-phenylethene-1,2diyl). While the use of standardized names should be encouraged, the promotion of a shared data ecosystem must have a strategy for managing the multitude of naming conventions (e.g., mapping methodologies such as ChemProps). ${ }^{46}$

Another challenge in the development of a shared language for polymers is their stochastic nature, which means that traditional forms of line notation such as SMILES strings fail to capture the full structural complexity. While the identity of the monomer is sometimes sufficient to allow for modeling of polymer properties with some success, ${ }^{47}$ higher-order complexities such as sequence and topology often have a dramatic effect. Certain developments have been made to capture some elements of polymer structure, such as the introduction of BigSMILES as machinereadable line notation, ${ }^{48}$ but additional developments are needed. Advances on this front are necessary to empower machine-learning tools to predict metrics by using polymer descriptors, allowing for further focus on research and analysis.

To improve the understanding and reuse of data, they should be presented not only using welldefined language but also with sufficient completeness. Metadata such as experimental parameters should be described in an established format, and the raw data used for processed data should be included and available. One avenue towards implementation of this strategy would be for funding agencies and journals to require the submission of original data in wellstructured files. In addition, more thorough undergraduate and graduate education concerning data best practices would serve to produce a generation of scientists prepared to navigate the increasingly data-heavy scientific climate.

\footnotetext{
${ }^{46} \mathrm{Hu}$, B. Y.; Lin, A. Q.; Brinson, L. C. "ChemProps: A RESTful API enabled database for composite polymer name standardization." Journal of Cheminformatics 2021, 13. http://doi.org/10.1186/s13321-021-00502-6.

${ }^{47}$ Le, T.; Epa, V. C.; Burden, F. R.; Winkler, D. A. "Quantitative Structure-Property Relationship Modeling of Diverse Materials Properties." Chemical Reviews 2012, 112, 2889-2919. http://doi.org/10.1021/cr200066h.

${ }^{48}$ Lin, T. S.; Coley, C. W.; Mochigase, H.; Beech, H. K.; Wang, W. C.; Wang, Z.; Woods, E.; Craig, S. L.; Johnson, J. A.; Kalow, J. A.; Jensen, K. F.; Olsen, B. D. "BigSMILES: A Structurally-Based Line Notation for Describing Macromolecules." ACS Central Science 2019, 5, 1523-1531. http://doi.org/10.1021/acscentsci.9b00476.
} 
(4b) How might we curate and use reliable data sets, assign quality measures, account for evolution in quality over time in sustainable polymer research to achieve accessible databases based on descriptive, searchable parameters?

The production of well-defined, reliable data is only one piece of the puzzle, as the larger goal is the creation of high-quality databases that enable accessibility and reusability. While attempts to assemble polymer properties into a single source have been made, ${ }^{49,50,51}$ we envision a broader, more open database, with a specific focus on the understanding and development of sustainable polymers. Such a database would require management by a centralized organization such as a data institute, which would provide the necessary infrastructure for cross-institution collaboration. A model for one such database is the Protein Data Bank (PDB). ${ }^{52}$

One issue in curating polymer data is the assessment of their quality and the determination on which to include, especially when considering previously generated datasets that may be incompletely described or measured with outmoded techniques. A decision must be made as to whether the inclusion of previously collected data merits the logistical challenge it presents; it is important, however, that these databases be considered as ever-evolving resources, with data being updated over time as understanding evolves. The implementation of a shared language and more complete data description, as discussed previously, would encourage more faith in data quality.

(4c) How might we encourage, incentivize, and streamline sharing between various academic and industrial sources, while navigating issues of confidential information, intellectual property, and attribution?

Another barrier towards the development of widely accessible databases is that, even when useful, well-described data are being produced, there may be no system nor any incentives for sharing them. Indeed, in the case of industry, there may be competitive disadvantages towards providing scientific data to a wider audience. There is also a potential conflict with base material providers (i.e., producers of polymers) sharing data about their products with the various and diverse "converters" that generate products who may be less inclined to share proprietary data. However, in the interest of avoiding duplicate efforts and advancing the cause of sustainable polymers with the greatest efficiency, we must find ways around this roadblock.

\footnotetext{
${ }^{49}$ Otsuka, S.; Kuwajima, I.; Hosoya, J.; Xu, Y.; Yamazaki, M. PoLylnfo: Polymer Database for Polymeric Materials Design. In 2011 International Conference on Emerging Intelligent Data and Web Technologies; 2011 ; pp 22-29. https://doi.org/10.1109/EIDWT.2011.13.

${ }^{50}$ Kim, C.; Chandrasekaran, A.; Huan, T. D.; Das, D.; Ramprasad, R. "Polymer Genome: A Data-Powered Polymer Informatics Platform for Property Predictions." Journal of Physical Chemistry C 2018, 122, 17575-17585. http://doi.org/10.1021/acs.jpcc.8b02913.

51 Ma, R. M.; Luo, T. F. "PI1M: A Benchmark Database for Polymer Informatics." Journal of Chemical Information and Modeling 2020, 60, 4684-4690. http://doi.org/10.1021/acs.jcim.0c00726.

52 Burley, S. K.; Berman, H. M.; Kleywegt, G. J.; Markley, J. L.; Nakamura, H.; Velankar, S. "Protein Data Bank (PDB): the single global macromolecular structure archive." Protein Crystallography 2017, 627-641. https://doi.org/10.1007/978-1-4939-7000-1_26.
} 
To this end, there are two challenges to address: the lack of a system and the lack of incentives. For the former, there currently exist many venues for the sharing of scientific results; in particular, peer-reviewed publications can be harnessed to enforce better data practices, as previously discussed. Requiring improved data presentation and accessibility upon publication serves the two-fold purpose of both providing a system for data sharing and incentivizing that sharing within the academic community due to the existing value placed on publication.

Data sharing amongst academics can be further incentivized by tracking data citations and transitive credit, and innovative data practices could be more strongly considered as part of tenure processes and promotions. Furthermore, the identification of sustainability questions that can be addressed via data sharing would allow for this form of collaboration to be directly incentivized via funding from federal agencies. Government incentives could be additionally used to balance the risk associated with sharing and the added value from sustainability-related initiatives, i.e., the encouragement of triple bottom line thinking. Further study will be necessary to understand the most effective strategies for incentivizing data sharing across all stakeholders.

Key Takeaways

- Key priorities must be identified for the field of sustainable polymers, as well as relevant metrics, to focus data accumulation on the most urgent issues.

- A centralized, well-managed database is essential to avoid redundancy and make data accessible for both human and machine learning efforts alike.

- Data sharing will need to be incentivized for researchers within academia, industry, and the public sector, which will require buy-in from various stakeholders.

\section{(5) Sustainable polymer communication, policy, and education through the development of universal language and shared understanding}

Developing a framework in sustainability where all stakeholders communicate through the same universal language is a challenging, but necessary process to reach a common understanding. There are factors to consider when creating a common ground in language and understanding of sustainable polymers: (i) differing scales (e.g., global vs. local); (ii) a basis in facts and data that is not driven by unsubstantiated perceptions; (iii) the diverse stakeholders from different sectors, including the scientific community in industry and academia, the public, policymakers, and federal institutions

Addressing sustainability at the global level requires achieving success at the local scale to set a leading example where others can follow. The diversity in geographical regions and cultural attitudes has an impact on the ability to not just create a universal language but encourage its adoption. Accessibility will play a major role in ensuring that the policies that come with creating this universal language are respected by different nations. At the same time, avoiding an overly simplistic policy is essential in preventing unsustainable behavior and mitigating the damage from misinformation and narratives driven by emotion and less on facts. A starting point in 
handling this Herculean task will involve three questions centered on using developments in science and technology to shape policy, integrating sustainability into education and training, and facilitating translation of developments across disciplines and cultures to enable an effective diverse interdisciplinary and inter-organization workforce. Inspired by the materials science and engineering tetrahedron, a paradigm is illustrated in Figure 5-1 that connects four interconnected components essential for working towards a framework of sustainable production.

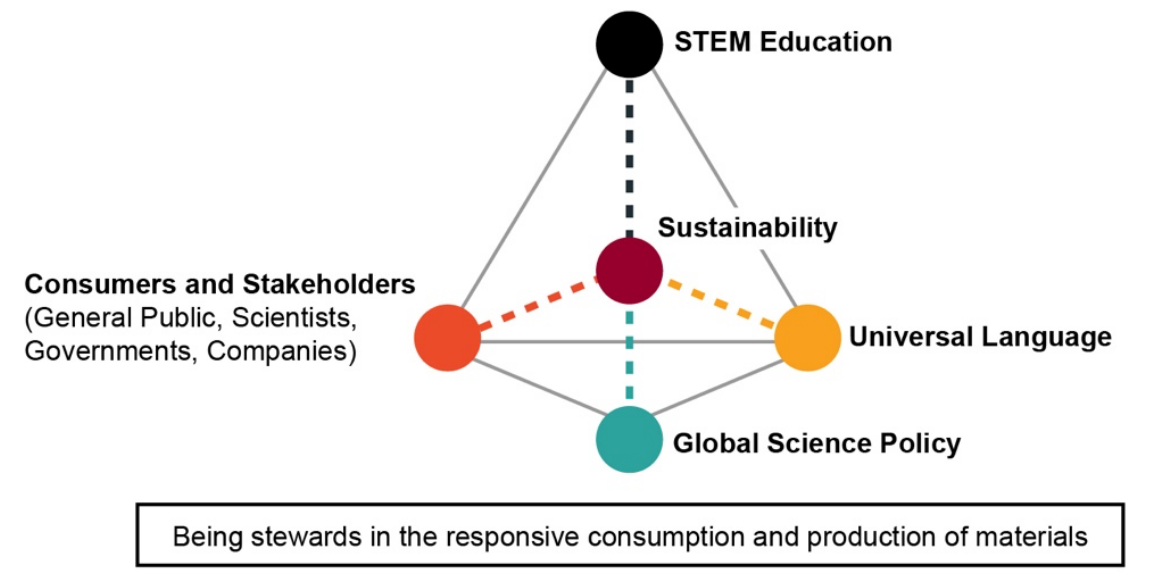

Figure 5-1. A proposed sustainable materials paradigm.

(5a) How might we use science and technology developments to influence and shape global public policy and regulatory structures around product sustainability and stewardship, and similarly, better understand policy and regulatory frameworks to inform scientific and technology innovations?

Creating policy on product sustainability needs to be grounded in scientific fact and the underlying data must be statistically representative. ${ }^{53}$ Showcasing examples of how science has benefited global communities is a start in influencing policymakers within the US and other countries. For example, "Science Can Change the World" is a video designed by DSM as a tribute to scientists who developed innovative contributions to tackling global issues surrounding food, disabilities, using waste feedstocks to create materials, and mosquito-borne diseases. ${ }^{54,55,56,57}$ There are numerous examples of scientific innovations that have contributed to the benefit of society in numerous ways across different sectors. Raising awareness around using exemplary cases is very much needed to convince the public and policymakers why science should be considered when approaching regulations and laws on product sustainability.

53 Vorpahl, S.; Montoni, N., Science and Public Policy. American Chemical Society: 2020. https://pubs.acs.org/doi/book/10.1021/acs.infocus.7e4005.

54 https://www.hugokeijzer.com/science-can-change-the-world (accessed 19 July 2021)

${ }^{55}$ For an example on algae that sequester carbon dioxide see: https://energaia.com/ (accessed 19 July 2021)

${ }^{56}$ For an example on sustainable aquaculture practices see: $\underline{\text { https://www.aquaculturealliance.org/advocate/just- }}$ add-sun-behind-energaias-evolving-spirulina-strategy/ (accessed 19 July 2021)

${ }^{57}$ For an example on edible water capsules see: Patel, P. "Edible Packaging." ACS Central Science 2019, 5, 1907-1910. http://doi.org/10.1021/acscentsci.9b01251. 
Inspiration in developing policy can be garnered from existing global commitments. The Paris Agreement, Montreal Protocol, and Kyoto Protocol represent committed global efforts to tackle climate change, depletion of the ozone layer, and reduction of greenhouse gas emissions, respectively. ${ }^{58,59,60}$ A similar obligation is necessary to achieve sustainable production of materials. UN Sustainable Development Goal (SDG) 12: Responsible Consumption and Production can be used as a model for constructing global public policy. ${ }^{61}$ Selected targets to be reached by 2030 for SDG 12 include: (i) efficient management and use of natural resources; (ii) management of waste in food and chemicals, (iii) reduced generation of waste using prevention, reduction, recycling, and reuse, (iv) encouragement of the adoption of sustainable practices and information in companies, and ( $v$ ) global accessibility of information and awareness of sustainability development. All these elements could be part of the Polymer Sustainability Roadmap discussed in Section 3.

Reduction of waste has been exemplified through the Beyond Benign's Green Chemistry Commitment and the U.S. Plastics Pact. ${ }^{62,63}$ Even though the US does not have federal regulations limiting single-use plastics, several states have enacted restrictions through either bans or fees and taxes. ${ }^{64}$ Directive (EU) $2019 / 904$ is a recent commission of guidelines for single-use plastics ban. ${ }^{65}$ This directive is aligned with several aspects of UN SDG 12. US governmental agencies, such as the Environmental Protection Agency (EPA) has provided incentives on adopting sustainable practices exist through funding projects on green chemistry for academic research and small businesses, which many have been recognized with awards. ${ }^{66}$ Ideas on creating policy on sustainable production can potentially incorporate those seen in the regulation of medical waste, which is heavily monitored by the EPA, Occupational Safety and Health Administration, and many others. ${ }^{67}$

(5b) How might we broadly integrate sustainability across the entire spectrum of education and training, spanning the general public to practitioners of polymer design, discovery, and development?

As emphasized throughout this document, the incorporation of sustainability in education and training practices is important to drive understanding and develop innovative concepts. Creating

\footnotetext{
58 https://unfccc.int/process-and-meetings/the-paris-agreement/the-paris-agreement (accessed 19 July 2021)

59 https://www.state.gov/key-topics-office-of-environmental-quality-and-transboundary-issues/the-montrealprotocol-on-substances-that-deplete-the-ozone-layer/ (accessed 19 July 2021)

60 https://unfccc.int/process-and-meetings/the-kyoto-protocol/what-is-the-kyoto-protocol/kyoto-protocol-targetsfor-the-first-commitment-period (accessed 19 July 2021)

${ }^{61}$ https://www.un.org/sustainabledevelopment/sustainable-consumption-production/ (accessed 19 July 2021)

62 https://www.beyondbenign.org/he-green-chemistry-commitment/ (accessed 19 July 2021)

63 https://usplasticspact.org/ (accessed 19 July 2021)

64 https://www.ncsl.org/research/environment-and-natural-resources/plastic-bag-legislation.aspx (accessed 19 July 2021)

65 https://eur-lex.europa.eu/legal-content/EN/LSU/?uri=CELEX:32019L0904 (accessed 19 July 2021)

66 https://www.epa.gov/greenchemistry/green-chemistry-challenge-winners (accessed 17 June 2021)

${ }_{67}$ Banks, M.; Metz, M.; Smyth, D. S. "The Sustainability Challenges Facing Research and Teaching Laboratories When Going Green." Environment 2020, 62, 4-13. http://doi.org/10.1080/00139157.2020.1708166.
} 
a pipeline for students in K-12 and college, as well as people beyond school in a wide range of communities, to learn and apply sustainability concepts is required to create a diverse workforce that understands the consequences of plastic and other types of waste. Education in sustainability provides an opportunity to modify our current teaching methods to better connect with students over traditional-based styles that have limited reach. Exposure to sustainability provides a connection to real-world problems, particularly with social and environmental justice, that can better engage students and facilitate effective problem-solving in their future work. The resources and infrastructure discussed in this section are potential answers in addressing how to integrate sustainability on a broader level.

Making room for sustainability as a teaching subject in a crowded curriculum is a difficult challenge, as fundamental topics need to be covered within a finite amount of time. Additionally, it is important to balance curriculum and training exercises without compromising the necessary skills that need to be taught. Knowing the set guidelines and established curriculum, especially in terms of how broad, multidimensional, and cross-disciplinary they will help in finding where sustainability can be placed. Supplementing core topics with sustainability has been achieved through the efforts of initiatives such as Green Chemistry Education. ${ }^{68}$ While sustainable concepts and green chemistry have been primarily showcased in upper level elective ${ }^{69,70}$ and organic chemistry lab courses, ${ }^{71,72,73,74}$ efforts have been made to expand the curriculum into

\footnotetext{
${ }^{68}$ Aubrecht, K. B.; Bourgeois, M.; Brush, E. J.; Mackellar, J.; Wissinger, J. E. "Integrating Green Chemistry in the Curriculum: Building Student Skills in Systems Thinking, Safety, and Sustainability." Journal of Chemical Education 2019, 96, 2872-2880. https://doi.org/10.1021/acs.jchemed.9b00354.

${ }^{69}$ Kennedy, S. A. "Design of a Dynamic Undergraduate Green Chemistry Course". Journal of Chemical Education. 2016, 93, 645-649. https://doi.org/10.1021/acs.jchemed.5b00432.

${ }^{70}$ O’Neil, N. J.; Scott, S.; Relph, R.; Ponnusamy, E. "Approaches to Incorporating Green Chemistry and Safety into Laboratory Culture". Journal of Chemical Education. 2021, 98, 84-91. https://doi.org/10.1021/acs.jchemed.0c00134.

${ }^{71}$ Wu, N.; Kubo, T.; Sekoni, K. N.; Hall, A. O.; Phadke, S.; Zurcher, D. M.; Wallace, R. L.; Kothari, D. B.; McNeil, A. J. "Student-Designed Green Chemistry Experiment for a Large-Enrollment, Introductory Organic Laboratory Course." Journal of Chemical Education 2019, 96, 2420-2425. https://doi.org/10.1021/acs.jchemed.9b00375.

72 O’Neil, N. J.; Scott, S.; Relph, R.; Ponnusamy, E. "Approaches to Incorporating Green Chemistry and Safety into Laboratory Culture." J. Chem. Educ. 2021, 98, 84-91. https://doi.org/10.1021/acs.jchemed.0c00134.

73 Gormong, E. A.; Wentzel, M. T.; Cao, B.; Kundel, L. N.; Reineke, T. M.; Wissinger, J. E. "Exploring Divergent Green Reaction Media for the Copolymerization of Biobased Monomers in the Teaching Laboratory." J. Chem. Educ. 2021, 98, 559-566. https://doi.org/10.1021/acs.jchemed.0c00688.

74 Lee, D. B. "Re-Casting Traditional Organic Experiments into Green Guided-Inquiry Based Experiments: Student Perceptions." Green Chem. Lett. Rev. 2019, 12, 107-116. https://doi.org/10.1080/17518253.2019.1609598.
} 
introductory ${ }^{75,76,77}$ and general chemistry courses. ${ }^{78,79,80}$ The lack of green chemistry content in organic chemistry textbooks has been explored and it has been suggested that connections to societal issues and sustainability contribute to the development of leadership skills. ${ }^{81}$ The existing literature in green chemistry can be used as a template to develop strategies to fully integrate sustainable polymers into the curriculum. The design elements used in capstone projects, which contribute tremendously to student development and creativity, should be considered for integrating sustainability concepts. ${ }^{82}$ Sustainability minors at the college undergraduate or even graduate levels could also be promoted and bolstered.

STEM outreach provided through supplemental instruction from workshops and programs developed by governmental bodies, research institutions, and non-profit organizations are ways to engage and expose K-12 students, their families, and instructors to sustainability. As an example, The NSF Center for Sustainable Polymers (CSP) has collaborated with 4-H in developing a science curriculum based on the Next Generation Science Standards to introduce K-8 grade students to the work of scientists and engineers in materials and the concepts of reuse, recycle, and reduce. ${ }^{83}$ Workshops have been developed that train high school teachers to incorporate green and sustainable chemistry into their classrooms. ${ }^{84}$ Several exercises utilized in the workshop were adapted from Beyond Benign, a non-profit organization focused on science education in sustainability that provides the necessary tools and training for educators to fully

\footnotetext{
${ }^{75}$ Klara, K.; Hou, N.; Lawman, A.; Wu, L.; Morrill, D.; Tente, A.; Wang, L. Q. "Developing and Implementing a Simple, Affordable Hydrogen Fuel Cell Laboratory in Introductory Chemistry." Journal of Chemical Education. 2014, 91, 19241928. https://doi.org/10.1021/ed4007875.

${ }^{76}$ Purcell, S. C.; Pande, P.; Lin, Y.; Rivera, E. J.; Latisha, P. U.; Smallwood, L. M.; Kerstiens, G. A.; Armstrong, L. B.; Robak, M. T.; Baranger, A. M.; Douskey, M. C. "Extraction and Antibacterial Properties of Thyme Leaf Extracts: Authentic Practice of Green Chemistry." J. Chem. Educ. 2016, 93, 1422-1427. https://doi.org/10.1021/acs.jchemed.5b00891.

77 Bopegedera, A. M. R. P.; Perera, K. N. R. "Greening" a Familiar General Chemistry Experiment: Coffee Cup Calorimetry to Determine the Enthalpy of Neutralization of an Acid-Base Reaction and the Specific Heat Capacity of Metals." J. Chem. Educ. 2017, 94, 494-499. https://doi.org/10.1021/acs.jchemed.6b00189.

${ }^{78}$ Armstrong, L. B.; Rivas, M. C.; Zhou, Z.; Irie, L. M.; Kerstiens, G. A.; Robak, M. A. T.; Douskey, M. C.; Baranger, A. M. "Developing a Green Chemistry Focused General Chemistry Laboratory Curriculum: What Do Students Understand and Value about Green Chemistry?" Journal of Chemical Education. 2019, 96, 2410-2419. https://doi.org/10.1021/acs.jchemed.9b00277.

${ }^{79}$ Klingshirn, M. A.; Spessard, G. O. "Integrating Green Chemistry into the Introductory Chemistry Curriculum." ACS Symposium Series 2009, 1011, 79-92. https://doi.org/10.1021/bk-2009-1011.ch005.

${ }^{80}$ Gron, L. U.; Bradley, S. B.; McKenzie, J. R.; Shinn, S. E.; Teague, M. W. "How to Recognize Success and Failure: Practical Assessment of an Evolving, First-Semester Laboratory Program Using Simple, Outcome-Based Tools." Journal of Chemical Education. 2013, 90, 694-699. https://doi.org/10.1021/ed200523w.

${ }^{81}$ Johnson, S.; Meyers, M.; Hyme, S.; Leontyev, A. "Green Chemistry Coverage in Organic Chemistry Textbooks." Journal of Chemical Education. 2020, 97, 383-389. https://doi.org/10.1021/acs.jchemed.9b00397.

82 Husanu, I. N. C.; Ertekin, Y.; Chiou, R.; Mauk, M. G. "The Contribution of Capstone Projects in Green/Renewable Energy Areas to Growth of the Engineering Curriculum in Global Sustainable Development." ASEE Annual Conference \& Exposition Conference Proceedings 2017, 2017-June. https://doi.org/10.18260/1-2--28951.

${ }^{83}$ www.4hpolymers.org/ (accessed 19 July 2021)

${ }^{84}$ Wissinger, J. E.; Knutson, C. M.; Javner, C. H. "Designing Impactful Green and Sustainable Chemistry Workshops for High School Teachers". ACS Symposium Series 2020, 1344, 1-14. https://doi.org/10.1021/bk-2020-1344.ch001.
} 
integrate green chemistry. ${ }^{85}$ The ChemAttitudes project collaboration between the American Chemical Society and the National Informal STEM Education Network is focused on building interest, relevance, and self-efficacy in chemistry for public audiences. ${ }^{86}$ This framework was incorporated in the Let's Do Chemistry Train-the-Trainer Workshop to improve chemistry outreach activities by providing strategies to better train others in designing activity content, format, and facilitation. It is worth exploring the learning framework and design-based research approach that ChemAttitudes employs for sustainable materials, which targets general audiences.

While major strides have been made to educate the youth, greater efforts are needed to target the public. One way of approaching this involves the utilization of, for example, TED talks. ${ }^{87}$ These videos can be very influential as they can connect scientists to a broader audience. Additionally, sports organizations, such as the International Olympic Committee (IOC) have used their influence to engage athletes and fans on sustainability topics. ${ }^{88}$ Olympic athlete Hannah Mills has used her sporting background as a platform to launch the Big Plastic Pledge to reduce the usage of plastics. ${ }^{89}$ Such sporting organizations have a strong global influence and can bring communities together to aspire for a greener world. For those who prefer self-teaching methods, the EPA has accessible online information for people to learn about the basics of green chemistry and the tools and resources available for creating sustainable communities. ${ }^{90,91,92}$

These examples illustrate how to bring to the fore sustainability across different spectrums. Figure 5-2 showcases how sustainability can easily be disseminated through such an interconnected network. The next potential step is to make sustainability a core subject area in education to extend its reach beyond more than the few interested individuals. Making sustainability a required learning subject will require a committed effort from federal and local governments, school districts, universities, and industry.

${ }^{85}$ www.beyondbenign.org (accessed 19 July 2021)

${ }^{86}$ www.nisenet.org/chemattitudes (accessed 19 July 2021)

${ }_{87}$ www.ted.com/talks/vivian tan are bio plastics a sustainable solution (accessed $19 \quad$ July 2021); www.ted.com/talks/andrea kruse bio based_plastics from_agricultural waste (accessed 19 July 2021)

88 https://olympics.com/ioc/sustainability (accessed 19 July 2021)

89 https://olympics.com/ioc/news/hannah-mills-sailing-towards-a-more-sustainable-future (accessed 19 July 2021)

90 https://www.epa.gov/greenchemistry/basics-green-chemistry\#ppa (accessed 19 July 2021)

91 https://www.epa.gov/smartgrowth/tools-and-resources-sustainable-communities (accessed 19 July 2021)

92 https://www3.epa.gov/carbon-footprint-calculator/ (accessed 19 July 2021) 


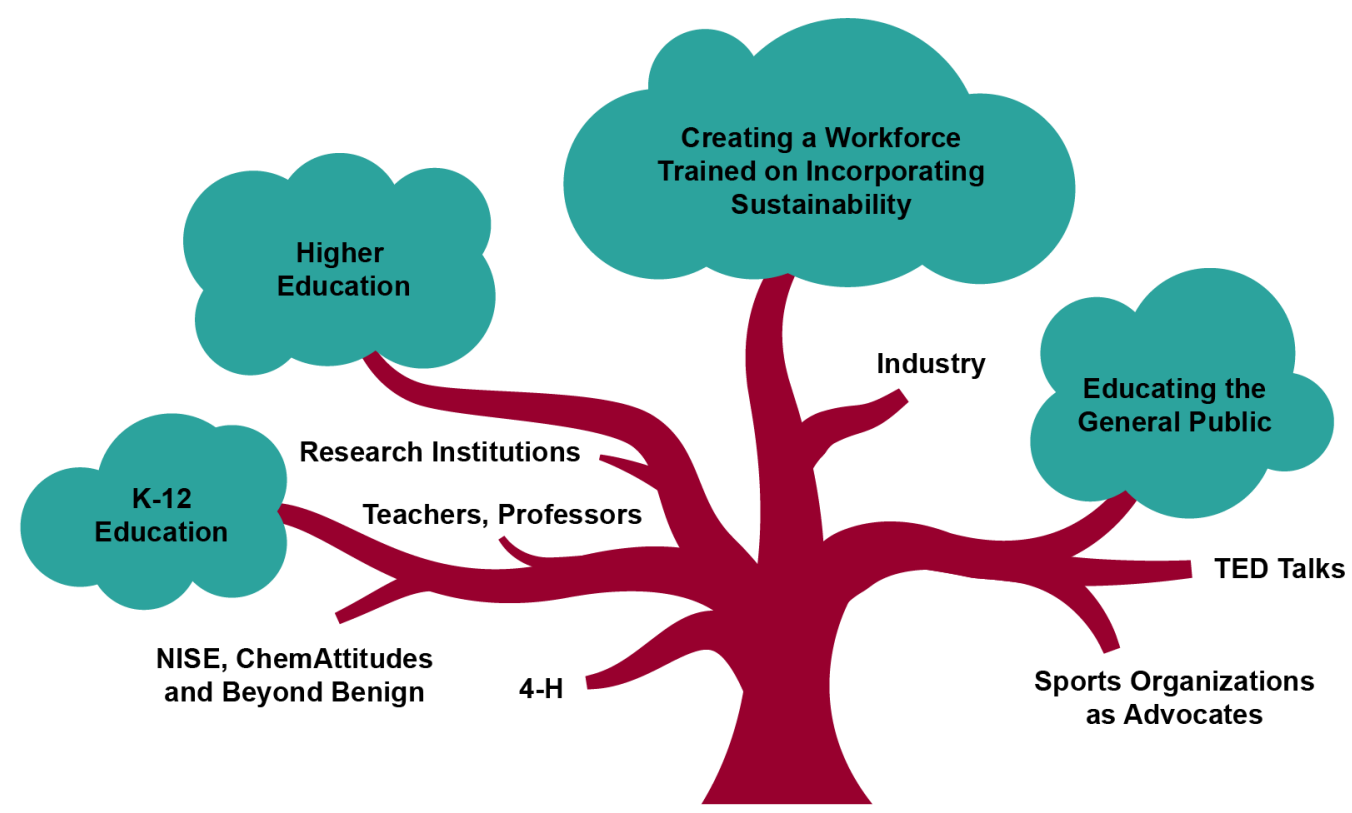

Increasing the Visibility of Sustainability by Making it a Core Learning Objective in Education

Figure 5-2. Increasing sustainable polymer visibility through cross-platform integration.

(5c) How might we establish a common sustainability language that facilitates understanding and translation of new technologies across disciplines and cultures and enables and sustains effective interdisciplinary and inter-organization teams?

Generating a system where the language of sustainability is universal across disciplines and institutions is critical. As alluded to earlier, having common definitions that can be shared and accepted by people from different backgrounds will help facilitate adoption of sustainable policies and practices by diverse communities. Besides defining sustainability in a general language accessible to everyone, it is just as important to involve stewardship where we take responsibility for the sustainability of the products we manufacture and introduce to society. Translation into native languages, global differences in socioeconomics, and accommodating the needs of habitants in each region need to be considered when adopting this universal language. The International Union of Pure and Applied Chemistry (IUPAC), the United Nations (UN), Sustainability reports from industry, and the NSF CSP have demonstrated the need to communicate in a common language.

The IUPAC, which is a non-governmental organization, is responsible for the creation and regulation of chemical nomenclature and terminology that is the common language for all sectors in chemical sciences. ${ }^{93}$ Using an IUPAC-like terminology system may be a useful approach in

93 iupac.org (accessed 19 July 2021) 
generating current definitions of common terms used to describe "sustainability", "circular", "recyclable", and "biodegradable". This approach will be potentially useful for labeling systems of materials, especially regarding what is considered recyclable. A clear labeling system that can be easily communicated to consumers is critical. The 17 UN SDGs is a great example of how to connect all stakeholders in the consumption of sustainable materials, as it addresses social and environmental issues at the global level. ${ }^{94}$ The Beyond the Classroom Webinar Series was created by the NSF Center for Sustainable Polymers to serves its members by increasing their knowledge on relevant research topics build a shared understanding with their interdisciplinary peers. ${ }^{95}$ This collective strategy can potentially help with the establishment of a community where science, industry, policy, and the general public can understand each other and thus assemble and collaborate in addressing sustainability. Key sustainable polymer terms that will need to be clearly defined for all consumers and stakeholders include closed-loop recycling, compostable plastic, and biodegradable plastic.

Key Takeaways

- Policy on sustainable production is needed and the level of commitment should be similar to the Paris Agreements, Montreal Protocol, and Kyoto Protocol.

- The topic of (polymer/plastic) sustainability should be a core topic taught and woven through curricula at all levels of education.

- A universal language for (polymer/plastic) sustainability which is accessible to everyone is needed.

(6) Societal adoption of sustainable polymers manufactured for in-use performance and circularity in terms of triple bottom line thinking: people, profit, planet

Adoption of sustainable polymers in place of currently unsustainable materials requires the participation of multiple stakeholders including end consumers, industry members, policymakers, and material scientists. In considering the obstacles to the widespread adoption of sustainable polymers, two sets of questions have been brought up repeatedly throughout this document. They must be answered for adoption to take place. The first set of questions challenges scientists and engineers to better design for sustainability at all parts of a product's life cycle. The second set is concerned with how usage of sustainable materials may be encouraged among end-users through changes in values and practices. Answering these questions leads to two conclusions. Sustainable materials should strive to attain price/performance ratios equal to or better than current materials to gain widespread adoption. Where obtaining this parity is not possible, it is imperative that public policies fill the gap. Note that seeking a balance between sustainability and profitability has long been recognized as a

94 https://sdgs.un.org/goals (accessed 19 July 2021)

95 https://csp.umn.edu/beyond-the-classroom/ (accessed 19 July 2021) 
laudable goal, and available frameworks could be built upon to address the plastics challenge. ${ }^{96}$ It is important that, through an effective combination of technology, policy, and education, we evolve towards an overarching culture that accepts lower profitability for the sake of a sustainable future.

(6a) How might we design economic viability, performance, and design for sustainability in terms offeedstock, use, and end-of-life, into sustainable polymer products from the cradle to the grave?

The challenge of designing for sustainability lies primarily in the responsibility of scientists and engineers from both industry and academia. Of the three criteria mentioned above, economic viability, performance, and sustainability over product lifetime, the last is the most difficult to evaluate and measure. In contrast, both performance and economic viability are quite straightforward, e.g., the desired modulus or desired price point. Sustainability, however, is difficult to quantify and can change over time as new feedstocks and consumer trends emerge. Over the manufacturing lifetime of a product, underlying production methods for raw materials may become sustainable, and so the goal of sustainability falls not just on ensuring that new products and materials are sustainable but also on continuing sustainable methods throughout the period the product is manufactured.

For sustainability to become a reasonable design parameter, universal metrics must be developed that, throughout a material's life, evaluate the waste generated, evaluate any benign products that might be generated through degradation, and quantify energy used in its creation. Food containers and plastic straws, for example, score poorly on this scale, with their single-use nature and short use lifetimes compared to their much longer lifetimes as waste. Other reusable alternatives score better but may have other adverse aspects (e.g., energy use), given they are more difficult to manufacture. Refinements on this strategy that also incorporate parameters such as greenhouse gas emissions and energy consumption have been put forward by several authors. ${ }^{97,98,99}$ The difficulties of determining such metrics have been emphasized in Section 4.

Quantifying sustainability is central to sustainable material and product development; note, however, that sustainability alone is not enough. Newly developed sustainable products must have the same or greater value to consumers. So long as the costs associated with unsustainable materials (e.g., landfilling, environmental impact) are externalized, while costs associated with sustainable materials (e.g., higher materials cost, inferior performance) are not, stakeholders in

\footnotetext{
96 "Good Chemistry for KAITEKI: A Challenge to a Sustainable, Healthy and Comfortable Society" Yoshimitsu Kobayashi, Hankyu Communications, 2011.

${ }^{97}$ Hasheminasab, H.; Gholipour, Y.; Kharrazi, M.; Streimikiene, D. "A novel Metric of Sustainability for petroleum refinery projects." Journal of Cleaner Production 2018, 171, 1215-1224. http://doi.org/10.1016/j.jclepro.2017.09.223.

${ }^{98}$ Sikdar, S. K. "On aggregating multiple indicators into a single metric for sustainability." Clean Technologies and Environmental Policy 2009, 11, 157-161. http://doi.org/10.1007/s10098-009-0225-4.

${ }^{99}$ Chong, Y. T.; Teo, K. M.; Tang, L. C. "A lifecycle-based sustainability indicator framework for waste-to-energy systems and a proposed metric of sustainability." Renewable \& Sustainable Energy Reviews 2016, 56, 797-809. http://doi.org/10.1016/j.rser.2015.11.036.
} 
the consumer section will not adopt sustainable materials on a large scale. An example of this adoption failure is the compostable packaging introduced in 2010 for the Sun Chip product line. Unaccounted for in the release was that, by changing to the new biodegradable polymer, the new material had a higher glass transition which in turn caused excessive crinkling noise when the packaging was opened or disturbed. As minor as this issue would seem, consumers rejected the new packaging. ${ }^{100}$ The lesson here is clear, consumers have expectations about product cost and performance and are not yet prepared to sacrifice these for sustainability. Sustainable alternatives must match both the price point and performance of unsustainable current-use materials if they are to be adopted. This fact influences and leads naturally to the second question for societal adoption of sustainable materials.

(6b) How might we understand and apply what is required to shift societal values and behaviors towards full adoption of sustainable materials and practices?

As demonstrated by the anecdote in the prior section, societal adoption of sustainable alternatives relies upon alternatives of equal or better price and performance. When these alternatives exist, adoption is relatively easy to achieve, but we must accept that this scenario will not always be the case. While science and engineering have provided a multitude of materials, with polymers offering a wide selection of tunable properties, in some cases the sustainable alternative may not exist, particularly at an economic value that equals current options. In these scenarios, it falls to government and policymakers to introduce means to provide incentives that promote the use of sustainable materials; when cost-effective alternatives do not exist, policy provides a means to produce change.

As an example, consider plastic bags and straws, which often end up as plastic waste. Consumers were accustomed to the tactile properties and stability of plastic straws, and alternatives such as paper and metal either may not be as sound, give a different drinking experience, or require the consumer to output capital or extra work to acquire them. Thus, the alternatives existed but were not being used. The solution arrived at for various municipalities was to ban these items, thereby forcibly phasing out their use. ${ }^{101}$

Similar stories can be found regarding plastic bags where, to curb their use, municipalities have implemented a bag tax to reduce consumption. While taxes seem to have universally reduced disposable plastic bag waste, in some respects these taxes have not entirely had their desired

\footnotetext{
${ }^{100}$ Evans, D. M.; Parsons, R.; Jackson, P.; Greenwood, S.; Ryan, A. "Understanding plastic packaging: The co-evolution of materials and society." Global Environmental Change 2020, 65, 102166. https://doi.org/10.1016/j.gloenvcha.2020.102166.

${ }^{101}$ Schnurr, R. E. J.; Alboiu, V.; Chaudhary, M.; Corbett, R. A.; Quanz, M. E.; Sankar, K.; Srain, H. S.; Thavarajah, V.; Xanthos, D.; Walker, T. R. "Reducing marine pollution from single-use plastics (SUPs): A review." Marine Pollution Bulletin 2018, 137, 157-171. http://doi.org/10.1016/j.marpolbul.2018.10.001.
} 
effect. ${ }^{102,103}$ They are successful in lowering disposable plastic bag usage but have led to increased consumption of reusable bags made from other materials, which may in some cases have a greater negative impact on the environment. Consumer education will clearly play a critically important role in future sustainability efforts.

Participation in programs geared toward reuse and recycling also offers a cautionary tale. Even in markets where recycling is available and accessible, only a small percentage of plastic is recycled. This failure is due to two factors: a lack of user participation and a failure of industry to account for and match the need for recycling. Both these issues must be rectified to achieve sustainable adoption. There is clearly a need for data on the fate of plastics, including plastic bags of bottles, and a need for "fingerprinting" plastic products in a simple and inexpensive manner, thereby providing a tool to track their use (see Section 4).

The issue with implementing policies that incentivize sustainability by moving externalities to the end product users is that they bear the economic responsibility. This challenge is the critical area where societal change is necessary to implement policies that move towards sustainable materials and practices. Promoting sustainability as a worthwhile goal will require a highly multidisciplinary team of scientists, marketers, educators (as detailed in Section 5), and policymakers. Inspiration can be found in prior environmental and conservation movements. Examples include the establishment of the national park service under President Theodore Roosevelt and the establishment of the EPA under President Richard Nixon. In these cases, public opinion (Roosevelt's popularity) and influential events (the burning of the Cuyahoga river) pushed these policies through the bureaucratic processes. Such moments are rare and we, as people interested in sustainability, must be prepared to seize them.

Key Takeaways

- New sustainable materials must be equal to or better in performance and price for widespread adoption.

- Where no such alternative is available, policies are required to incentivize a transition.

\footnotetext{
102 Ritch, E.; Brennan, C.; MacLeod, C. "Plastic bag politics: modifying consumer behaviour for sustainable development." International Journal of Consumer Studies 2009, 33 (2), 168-174. http://doi.org/10.1111/i.14706431.2009.00749.x.

${ }^{103}$ https://plasticpollutioncoalitionresources.org/wpcontent/uploads/2017/03/Effectiveness of plastic regulation around the world 4 pages.pdf. (accessed 19 July 2021)
} 


\section{Conclusions and Outlook}

Sustainable polymers must be the future. Integrated efforts across many sectors and by all stakeholders are needed to realize the broad implementation of sustainable polymers posthaste. Advances in modeling and computation, coupled to new high throughput synthesis and characterization techniques, offer considerable promise for development of a new generation of polymeric materials that exhibit superior performance and whose environmental impact is taken into consideration throughout the design process and through the full life cycle of the material. The Materials Genome Initiative (MGI) has provided an effective framework for rapid development of materials. The original goal of that initiative was to decrease the time and cost of the materials discovery to deployment process. In the case of plastics and the environmental threat they represent, accelerating that process requires that the MGI framework be expanded in several ways to ensure rapid commercial adoption of new sustainable polymers, as well as that for entire sustainable systems that fully include end of use considerations. In particular, beyond a material's performance, the roles of ease of synthesis, ease of degradation, ease of upcycling, policy and cost must all be considered early in the design process.

New computational methods, along with the corresponding publicly available and wellmaintained software, must be developed. The aim of such tools should be that of predicting equilibrium and non-equilibrium properties and the role of processing on such properties, as well as chemical stability, toxicity, or degradation pathways. Similarly, well integrated, comprehensive data sets, and the corresponding databases are needed. There is a widespread consensus that, in the area of polymers, the amount and the breadth of available experimental and computationally generated data are insufficient and inadequate for the purposes of accelerating the discovery and translation of a new generation of sustainable plastics. Not only are more thermophysical property data needed; information related to the composition of polymers, including common additives, and the fate of different types of plastics in the environment, should be incorporated into such accessible and useable databases.

Plastics are ubiquitous, and a central challenge in the general area of plastics, sustainability, and environmental impact, is the fact that a wide range of stakeholders must come together to find solutions, fast. Users of plastics must specify minimal requirements, producers of plastics must develop the means to meet product and societal demands, environmental experts and government agencies must develop acceptable standards, industry and academia must develop new materials systems, and economists and policy experts must work along all these groups to ensure that such sustainable polymer systems are adopted. Getting these stakeholders to work collaboratively requires that a comprehensive roadmap be developed, with clear and agreed upon goals and a timeline associated with them. A systems-level approach is needed for such a roadmap to strike a balance between needs, benefits and impact, and have a net positive effect on the planet. Without roadmaps, it is difficult to arrive at (and enforce) effective, dynamic policies. An overarching organization of efforts is desperately needed that brings together representatives from each community of stakeholders to align expectations and chart a course forward for meaningful systemic change to our current linear plastics modus operandi. 
Moreover, embracing a system level view as far towards the beginning of the research pipeline will be ideal for integration of sustainability principles into early-stage research and design.

Similarly, it is important that these efforts be accompanied by educational and outreach programs at all levels. Educational and outreach campaigns should highlight the benefits that plastics provide in multiple domains, and at the same time expand on the environmental consequences that the current generation of materials presents. Responsible use of resources, be it avoiding the single use of plastics, or developing more mindful attitudes towards conservation and recycling, are concepts that must be more widely engrained in every aspect of education. They should also be of primary consideration as new products are introduced into the market. New generations of scientists and engineers should be exposed as part of their training to foundational concepts of sustainability, including the role of policy and governance. As they join the workforce, they will be able to deploy those concepts in real world applications.

Awareness of many of the issues raised in this report is rapidly growing. With federal funding, new research centers have been established to develop a new generation of sustainable polymeric materials, ${ }^{104}$ some of them focused on strategies based in biology. Focused roadmapping exercises by industry and government are starting to emerge. ${ }^{105}$ Awareness of the environmental impact of plastics is growing but given the magnitude of the challenge, much more is needed. Currently, hundreds of millions of tons of plastics are produced every year, and a corresponding amount of plastic waste are generated annually. The case for creating new materials, systems, infrastructure, and policies that lead to a reduction and elimination of that waste is compelling. This report has identified and summarized several key findings that, under the framework of the MGI, could lead to impactful solutions.

\footnotetext{
${ }^{104}$ For example, recent coordinated efforts include: BioPacific MIP (https://biopacificmip.org/ accessed 19 July 2021), BioMADE (https://www.manufacturingusa.com/institutes/biomade accessed 19 July 2021), and ReMADE (https://remadeinstitute.org/ accessed 19 July 2021).

105 For example: https://ebrc.org/focus-areas/roadmapping/roadmap-for-materials-science-engineering-biology/ accessed 19 July 2021; https://www.energy.gov/plastics-innovation-challenge/downloads/plastics-innovationchallenge-draft-roadmap-and-request accessed 19 July 2021; https://usplasticspact.org/roadmap-reader/ accessed 19 July 2021
} 
Appendix 1 - Square Table Participant List

\begin{tabular}{|c|c|c|}
\hline Name & Contact e-mail & Institution \\
\hline \multicolumn{3}{|l|}{ Leaders } \\
\hline Juan de Pablo & depablo@uchicago.edu & University of Chicago \\
\hline Marc Hillmyer & hillmyer@umn.edu & University of Minnesota \\
\hline \multicolumn{3}{|l|}{ Facilitators } \\
\hline Heather Crews & hcrews@uchicago.edu & University of Chicago \\
\hline Toby Scott & toby.scott@knowinnovation.com & Knowlnnovation \\
\hline Emma Skipper & emma.skipper@knowinnovation.com & Knowlnnovation \\
\hline \multicolumn{3}{|l|}{ Academia } \\
\hline Ben Blaiszik & blaiszik@uchicago.edu & University of Chicago \\
\hline Jeffrey Buenaflor & buena007@umn.edu & University of Minnesota \\
\hline Daphne Chan & chanx256@umn.edu & University of Minnesota \\
\hline Geoff Coates & gc39@cornell.edu & Cornell University \\
\hline Jennifer Dunn & jennifer.dunn1@northwestern.edu & Northwestern University \\
\hline Ming Han & hanmingcr@uchicago.edu & University of Chicago \\
\hline Nick Jackson & jacksonn@illinois.edu & Univ. of Illinois, UC \\
\hline Julia Kornfield & jak@cheme.caltech.edu & Caltech \\
\hline Lashanda Korley & Ikorley@udel.edu & University of Delaware \\
\hline Jodie Lutkenhaus & jodie.lutkenhaus@tamu.edu & Texas A\&M University \\
\hline Heather Maynard & maynard@chem.ucla.edu & UCLA \\
\hline Josh Mysona & jmysona@uchicago.edu & University of Chicago \\
\hline Brad Olsen & bdolsen@mit.edu & Mass. Inst. of Tech. \\
\hline Phil Rauscher & pmrauscher@uchicago.edu & University of Chicago \\
\hline Theresa Reineke & treineke@umn.edu & University of Minnesota \\
\hline Robert Riggleman & rrig@seas.upenn.edu & University of Pennsylvania \\
\hline Megan Robertson & mlrobertson@uh.edu & University of Houston \\
\hline Stuart Rowan & stuartrowan@uchicago.edu & University of Chicago \\
\hline Caitlin Sample & sampl128@umn.edu & University of Minnesota \\
\hline Ludwig Schneider & ludwigschneider@uchicago.edu & University of Chicago \\
\hline Katherine Shayne & kshayne1@uga.edu & University of Georgia \\
\hline Alejandro Strachan & strachan@purdue.edu & Purdue University \\
\hline Peter Voorhees & p-voorhees@northwestern.edu & Northwestern University \\
\hline \multicolumn{3}{|l|}{ Industry } \\
\hline Nicolas Cudre-Mauroux & nicolas.cudre-mauroux@solvay.com & Solvay \\
\hline Sharon Feng & s.feng@ppg.com & PPG \\
\hline Hart Haugen & hart.haugen@sherwin.com & Sherwin Williams \\
\hline Robert Kieschke & rrkieschke1@mmm.com & $3 \mathrm{M}$ \\
\hline Eric Klingenberg & eric.klingenberg@effem.com & Mars, Inc. \\
\hline Patrick Maestro & patrick.maestro@solvay.com & Solvay \\
\hline
\end{tabular}




\begin{tabular}{|c|c|c|}
\hline Jill Martin & jmmartin@dow.com & Dow Chemical \\
\hline Bryce Meredig & bryce@citrine.io & Citrine Informatics \\
\hline Jed Pitera & pitera@us.ibm.com & IBM \\
\hline Patrick Theofanis & patrick.theofanis@intel.com & Intel \\
\hline Judith van Gorp & judith.gorp-van@dsm.com & DSM \\
\hline \multicolumn{3}{|l|}{$\begin{array}{l}\text { National Labs/ } \\
\text { Government }\end{array}$} \\
\hline Evan Runnerstrom & evan.I.runnerstrom.civ@mail.mil & Army Research Office \\
\hline Kate Beers & kathryn.beers@nist.gov & NIST \\
\hline Massimiliano Delferro & delferro@anl.gov & Argonne National Lab \\
\hline Amalia Fernandez Panella & Amalia.Fernandez.Panella@moore.org & Moore Foundation \\
\hline Koushik Ghosh & kghosh@sandia.gov & Sandia National Lab \\
\hline Adam Jones & adam.jones@moore.org & Moore Foundation \\
\hline Jack Lewnard & jack.lewnard@hq.doe.gov & Department of Energy \\
\hline Jeff Spangenberger & jspangenberger@anl.gov & Argonne National Lab \\
\hline \multicolumn{3}{|l|}{$\begin{array}{l}\text { National Science } \\
\text { Foundation }\end{array}$} \\
\hline Peter Anderson & peanders@nsf.gov & NSF \\
\hline Bryan Boudouris & bboudour@nsf.gov & NSF \\
\hline Randy Duran & rduran@nsf.gov & NSF \\
\hline Bruce Hamilton & bhamilto@nsf.gov & NSF \\
\hline Alexios Klironomos & aklirono@nsf.gov & NSF \\
\hline Paul Lane & plane@nsf.gov & NSF \\
\hline Andrew Lovinger & alovinge@nsf.gov & NSF \\
\hline Shadi Mamaghani & smamagha@nsf.gov & NSF \\
\hline Linda Sapochak & Isapocha@nsf.gov & NSF \\
\hline Nora Savage & nosavage@nsf.gov & NSF \\
\hline John Schlueter & jschluet@nsf.gov & NSF \\
\hline Suk-Wah Tam-Chang & stamchan@nsf.gov & NSF \\
\hline
\end{tabular}




\section{Appendix 2 - Acknowledgements}

Thank you to all the participants who made the time to contribute to this important Square Table effort. Funding for this Square Table was provided by the National Science Foundation (DMR2127823). We thank Toby Scott and Emma Skipper from Knowlnnovation for facilitating the Square Table discussion and the substantive preparatory work prior to the two-day live event. We thank Heather Crews for critical administrative support throughout. We are grateful to the NSF Center for Sustainable Polymers for contributions to the report. Thank you to John Beumer (beumer@umn.edu) for graphic design contributions. We also acknowledge the two home institutions of the organizers/leaders: The University of Chicago and The University of Minnesota. A special thank you to the postdoctoral researchers Dr. Jeffrey Buenaflor, Dr. Daphne Chan, Dr. Ming Han, Dr. Josh Mysona, Dr. Phil Rauscher, Dr. Caitlin Sample, and Dr. Ludwig Schneider who in addition to making important contributions to the report, played key roles in capturing content from the Square Table discussion and drafting sections of the report. 